\title{
Interpenetrating polymer network hydrogels using natural based dyes initiating systems: Antibacterial activity and 3D/4D performance
}

Hong Chen ${ }^{1,2}$, Christophe Regeard ${ }^{3}$, Hanène Salmi ${ }^{4}$, Fabrice Morlet-Savary ${ }^{1,2}$, Nicolas Giacoletto ${ }^{5}$, Malek Nechab ${ }^{5}$, Pu Xiao ${ }^{6 *}$, Frédéric Dumur ${ }^{5^{*}}$, Jacques Lalevée $1,2^{*}$

${ }^{1}$ Université de Haute-Alsace, CNRS, IS2M UMR 7361, F-68100 Mulhouse, France; jacques.lalevee@uha.fr

${ }^{2}$ Université de Strasbourg, F-67200 Strasbourg, France

${ }^{3}$ Université Paris-Saclay, CNRS- CEA, I2BC, 91198 Gif sur Yvette, France

${ }^{4}$ Université Paris-Saclay, CNRS, ICMMO- SM2ViE UMR 8182, 91405 Orsay cedex, France

5 Aix Marseille Univ, CNRS, ICR UMR 7273, F-13397 Marseille, France ; frederic.dumur@univ-amu.fr

${ }^{6}$ Research School of Chemistry, Australian National University, Camberra, ACT 2601, Australia; pu.xiao@anu.edu.au

* Corresponding author: jacques.lalevee@uha.fr (J. L.), frederic.dumur@univ-amu.fr (F.D.); pu.xiao@anu.edu.au (P. X.).

Abstract: In the past few decades, interpenetrating polymer network (IPN) hydrogels have attracted huge attentions due to their special cell-like structures, interpenetrating interface, and two-phase continuity, which has special synergistic effects on performance or function. In this work, eleven different dyes based on 1-aryl-3-(2,4,5trimethoxyphenyl)prop-2-en-1-one or 3-aryl-1-(3,4,5-trimethoxyphenyl)prop-2-en-1one were firstly synthesized, and combined with an amine (i.e. ethyl 1,4(dimethylamino)benzoate, EDB) and an iodonium salt (bis(4-tert-butylphenyl) iodonium hexafluorophosphate, Iod) (PISs) as the three-component photoinitiating systems (PIS) to induce both the free radical photopolymerization (FRP) of polyethylene glycol diacrylate (PEG-DA) and the cationic photopolymerization (CP) of 3,4-epoxycyclohexylmethyl-3',4'-epoxy-cyclohexane carboxylate (EPOX) under the irradiation of LED@405nm. The best candidates were selected from the proposed eleven different dyes to study the involved oxidation-reduction reaction mechanisms 
through several characterization techniques. The best candidates were also used to prepare PEG-DA/EPOX interpenetrated polymer networks (IPNs). More interestingly, the stable 3D patterns have been successfully produced through direct laser write (DLW) technology, meanwhile, the silver cations could also be reduced in situ into silver nanoparticles. Furthermore, 4D printing could be achieved since all the obtained 3D patterns exhibited reversible swelling properties and shape-memory effect caused by swelling and dehydration processes. Parallel to this, influence of AgNPs on the mechanical properties of the 3D-printed objects and on IPNs has also been systematically studied. Finally, the obtained IPN polymers containing silver nanoparticles (AgNPs) exhibited antibacterial activities for Gram-positive bacteria (e.g. Staphylococcus aureus) and Gram-negative bacteria (e.g. Pseudomonas aeruginosa).

Keywords: interpenetrating polymer network; silver nanoparticles; antibacterial materials; reversible shape-memory effect; 3D/4D printing.

\section{Introduction}

Interpenetrating polymer networks (IPNs) are not just a mixture of two or more preformed polymers, but it refers to a network comprising two or more partially interwoven polymer networks that are not covalently bonded to each other [1-5]. Compared with a single polymer network, interpenetrating polymer network (IPN) hydrogels rely on the interweaving of multiple networks to form a special cell-like structure, interpenetrated interface and two-phase continuity, which greatly improves their physical and chemical properties or functions [6-10]. Due to the incompatibility of the multiple preformed polymers that make up the IPN, IPN polymers retain the characteristics of a single network. In addition, as the continuity and synergy of the two phases, their properties are homogenized and the final polymer exhibit unique properties that are different from those of the two polymers considered separately, which greatly broadens their scope of applications [11-16].

It is commonly known that PEG-DA allows the preparation of water compatible polymers is a water-soluble polymer. PEG-DA can exhibit a wide range of molecular weight (from several hundreds to tens of thousands), varying the scope of applications 
of the generated polymers is polymer. Owing to the low hydrogel cytotoxicity and good biocompatibility, hydrogels have been widely used in the fields of medical biology, pharmaceutical materials, etc [17-23]. However, polymer materials prepared with pure low molecular weight PEG-DA have poor rigidity and toughness, so that this polymer can be easily broken. Therefore, another flexible polymer network can be introduced into the rigid polymer network of PEG-DA to prepare IPN hydrogels and enhance the rigidity and toughness of the polymer. As previous studies of our group have demonstrated that some dyes derived from natural plants can be used as photoinitiator, which can not only initiate free radical polymerizations (of PEG-DA for instance) but also induce the cationic polymerization of epoxides such as EPOX under visible light irradiation [24-27]. EPOX is a commonly used flexible monomer that can be introduced into PEG-DA as a second network to prepare IPN hydrogels [28-30].

In this study, we fabricated IPN hydrogels based on PEG-DA/EPOX using threecomponent photoinitiating systems. At the same time, Ag nanoparticles could be synthesized in-situ upon introduction of a silver salt. Briefly, eleven different dyes were synthesized and used as photoinitiating systems (PIS) in combination with Iod/EDB. The best candidates were then selected to prepare IPN hydrogels containing dense (FRP of PEG-DA) and loose networks (CP of EPOX). Markedly, compared to a single network hydrogel, the polymerization efficiency and the mechanical properties of IPN hydrogels have been significantly enhanced. Subsequent to their syntheses, the mechanical and chemical properties of IPNs were comprehensively studied. During the photopolymerization process, the silver cations could be reduced by the generated free radicals into AgNPs that are evenly distributed into the hydrogels, what provides a new strategy for the preparation of antibacterial materials. Finally, the newly proposed photoinitiating systems were successfully used to prepare 3D patterns with reversible swelling properties and shape-memory effects. Furthermore, performance differences between single networks and interpenetrating polymer networks with different ratios (3:7/5:5/7:3) containing or not AgNPs were also investigated. Finally, antibacterial properties of IPNs polymers containing silver nanoparticles (AgNPs) were also studied. 


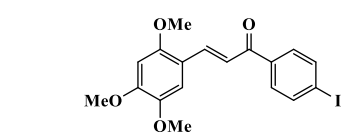

1

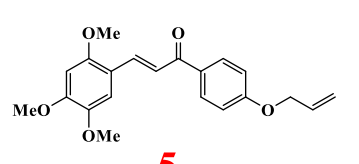

5

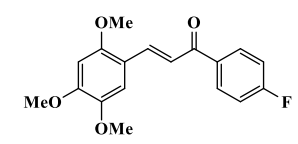

2

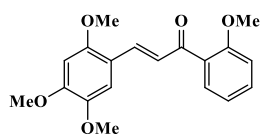

3

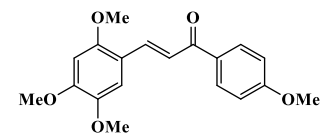

4
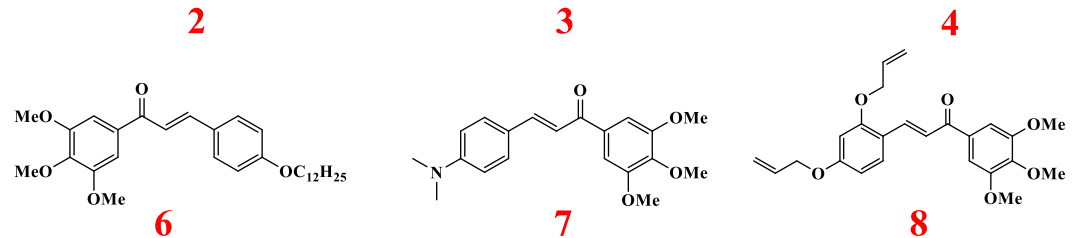

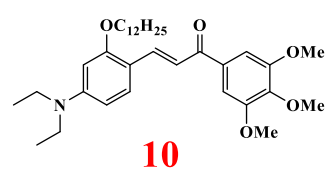

10

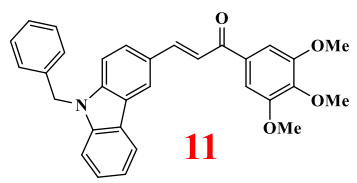

Scheme 1. Chemical structures of dyes 1-11.

\section{Materials and Methods}

\subsection{Material}

The 11 different chalcone-based dyes (1-11), whose molecular structures are shown in Scheme 1, were prepared according to the method reported in SI and used as photoinitiators. The monomers i.e. polyethylene glycol diacrylate (PEG-DA) and 3,4epoxycyclohexylmethyl 3,4-epoxycyclohexanecarboxylate (EPOX) were purchased from Sartomer-Europe and Allnex, respectively. The co-initiator bis-(4-tertbutylphenyl) iodonium hexafluorophosphate (Iod) and electron donor ethyl 4dimethylaminobenzoate (amine, EDB) were all purchased from Lambson Ltd. Chemical structures of these monomers and additives are presented in Scheme 2. Silver nitrate used as the precursor for AgNPs was purchased from Sigma-Aldrich (St. Louis, MO, USA). All other reagents were obtained from various suppliers and used as received. 


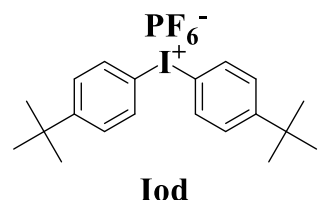

Monomers<smiles>C=CCOC(=O)OCCOC(=O)C=C</smiles>

PEG-DA<smiles>CCOC(=O)c1ccc(N(C)C)cc1</smiles>

EDB<smiles>O=C(OCC1CCC2OC2C1)C1CCC2OC21</smiles>

EPOX

Scheme 2. Chemical structures of the different monomers and additives used in this study.

\subsection{Photopolymerization ability of the proposed dye/Iod/amine-based PIS for the} PEG-DA/EPOX

Combination of the different dyes with Iod/amines were used as visible light PIS to study their initiating abilities for FRP and CP processes by real-time FTIR spectroscopy. In detail, the content of each component was optimized by comparing their solubility in the resin and photopolymerization efficiency, which were finally kept at $0.1 \mathrm{wt} \% / 1.5 \mathrm{wt} \% / 1.5 \mathrm{wt} \%(\mathrm{w} / \mathrm{w} / \mathrm{w}$, calculated from the weight of the resins that we used), respectively. All these dyes and additives are well dispersed into the resins (PEGDA/EPOX). After that, real-time FTIR spectroscopy (JASCO FTIR 4100) was used to continuously monitored the conversion of epoxy or acrylate functional groups under visible light irradiation (LED $405 \mathrm{~nm}, \mathrm{I}_{0}=110 \mathrm{~mW} / \mathrm{cm}^{2}$ ). During this process, the FRP of PEG-DA (thickness $\sim 20 \mu \mathrm{m} / 2 \mathrm{~mm}$ ) was carried out in the lamination conditions to reduce $\mathrm{O}_{2}$ inhibition, while the $\mathrm{CP}$ of EPOX (thickness $\sim 20 \mu \mathrm{m} / 2 \mathrm{~mm}$ ) was directly conducted in the air. The followed characteristic peak of EPOX is located around 790 $\mathrm{cm}^{-1}$ (thickness $\sim 20 \mu \mathrm{m}$ ) or $3700 \mathrm{~cm}^{-1}$ (thickness $\sim 2 \mathrm{~mm}$ ), while for PEG-DA, the characteristic peak is around $1600 \mathrm{~cm}^{-1}$ (thickness of about $\sim 20 \mu \mathrm{m}$ ) or $6160 \mathrm{~cm}^{-1}$ (thickness $\sim 2 \mathrm{~mm}$ ) [31]. IPN polymers were fabricated via the FRP of PEG-DA and the CP of EPOX. Briefly, we still use the proposed three-component PIS to initiate the photopolymerization reaction under visible light irradiation, in which the original single 
monomer (PEG-DA or EPOX, $100 \mathrm{wt} \%$ ) was replaced by mixed monomers (PEGDA:EPOX, $30 \mathrm{wt} \%: 70 \mathrm{wt} \%, 50 \mathrm{wt} \%: 50 \mathrm{wt} \%, 70 \mathrm{wt} \%: 30 \mathrm{wt} \%)$ in different proportions.

\subsection{Involved mechanism research}

\subsubsection{UV-Vis absorption properties of dyes and their photolysis process with}

\section{Iod/amine}

UV-vis absorption properties of these proposed dyes were studied in acetonitrile at the concentration of $1 \times 10^{-5} \mathrm{M}$ by a JASCO V730 spectrophotometer. After that, photolysis of dyes with Iod/amine and dyes alone were also studied in acetonitrile still using the JASCO V730 spectrophotometer, in which their concentrations were at $1 \times 10^{-}$ ${ }^{5} \mathrm{M}$ and $0.01 \mathrm{M}$, respectively [32].

\subsubsection{Fluorescence experiments of the dyes.}

A JASCO FP-6200 spectrofluorimeter was used to determine the fluorescence spectra of the dyes and the fluorescence quenching of them with Iod/amine, which was conducted in acetonitrile at the concentration of about $1 \times 10^{-5} \mathrm{M}$. The excitation wavelength was about $300 \mathrm{~nm}$.

\subsubsection{Electron Spin Resonance (ESR) Spin Trapping (ESR-ST) Experiments and the Redox Potentials of Dyes}

Radicals generated in $\mathrm{N}_{2}$ saturated tert-butylbenzene solutions containing the dyes and Iod/EDB $(0.1 \mathrm{wt} \% / 1.5 \mathrm{wt} \%)$ upon irradiation with sunlight were captured by PBN (0.25 wt\%) and analyzed with a X-band spectrometer (Bruker EMX-plus) at room temperature. The obtained ESR spectra were then simulated and analyzed by using the WINSIM software [33].

Redox potentials ( $\mathrm{E}_{\mathrm{ox}}$ and $\left.\mathrm{E}_{\mathrm{red}}\right)$ of chalcone-based dyes were determined by cyclic voltammetry that using tetrabutylammonium hexafluorophosphate as the supporting electrolyte in acetonitrile. Free energy changes ( $\Delta \mathrm{G}^{\mathrm{S} 1}{ }_{\mathrm{Iod}}$ or $\left.\Delta \mathrm{G}^{\mathrm{S} 1}{ }_{\mathrm{EDB}}\right)$ were calculated to prove the possibility of the electron transfer reactions between the dyes and the 
additives (Iod/amine) and supporting the photopolymerization processes observed experimentally. Redox potentials $\left(\mathrm{E}_{\mathrm{ox}}\right.$ and $\left.\mathrm{E}_{\mathrm{red}}\right)$ of the proposed dyes were determined by cyclic voltammetry in acetonitrile, while $\mathrm{E}^{*}$ corresponding to the excited state energy level of the dyes was determined from the crossing point of their UV-visible and fluorescence spectra, respectively.

\subsection{Preparation of the silver nanoparticles (AgNPs)}

\subsubsection{Preparation of AgNPs in DMF}

Silver nanoparticles were fabricated in DMF solution, which containing $0.5 \mathrm{wt} \%$ Dye/Iod/amine-based PIS $(0.1 \mathrm{wt} \% / 1.5 \mathrm{wt} \% 1.5 \mathrm{wt} \%)$ and $5 \mathrm{wt} \%-\mathrm{AgNO}_{3}$, upon irradiation with visible light LED@405nm [34].

\subsubsection{Preparation of AgNPs within polymers through in-situ Photopolymerization}

\section{Processes}

The dyes/Iod/amine (0.1wt\%/1.5wt\%/1.5wt\%)-based three-component PISs were completely dissolved in the mixed monomer (PEG-DA-EPOX). Then, $5 \mathrm{wt} \% \mathrm{AgNO}_{3}$ was added and the resulting solution was stirred at room temperature for $1 \mathrm{~h}$ to ensure the salt to be well-distributed into the formulation. After that, ultrasonic mixing for 5 minutes to evenly distribute the metal salt into the formulation was carried out. Finally, conversion profiles of the mixed monomers were followed by real-time FTIR spectroscopy, which not only enabled to determine the influence of the silver cations on the photopolymerization efficiency, but also enabled to prepare IPNs with uniformly distributed silver nanoparticles [34].

\subsection{Characterization of IPN Polymers.}

\subsubsection{TEM and SEM of AgNPs.}

Firstly, microscopic morphology of AgNPs formed in DMF as the solvent after 120 min of irradiation under a LED@405nm was examined by transmission electron microscope (TEM, FEI Quanta 250 FEG). Meanwhile, for AgNPs fabricated during the 
photopolymerization of IPNs, a scanning electron microscopy (SEM, FEI Quanta 250 FEG) was used to observe their morphologies [25,35].

\subsubsection{Dynamic time sweep rheology}

Photopolymerization processes of all the formulations were further detected through dynamic time-scanning rheology. In detail, $40 \mu \mathrm{L}$ of resin was dropped on a glass slide and then use a Haake-Mars Rotational Rheometer at a constant frequency of $10 \mathrm{~Hz}$ at $25^{\circ} \mathrm{C}[35]$.

\subsubsection{Tensile strength of polymers}

Firstly, various samples (length $4 \mathrm{~cm}$, width $2 \mathrm{~mm}$ and height $2.5 \mathrm{~mm}$ ) were prepared by using molds under the irradiation of visible light LED@405nm, and then the tensile strength of the prepared single network and interpenetrating network polymers were carried out by a Dynamometre Instron with a $100 \mathrm{~N}$ loading at a displacement rate of $0.1 \mathrm{~mm} / \mathrm{min}[36]$.

\subsection{Applications in 3D and 4D Printing.}

\subsubsection{Laser Write Experiments to prepare the Single and IPN polymers containing/without AgNPs}

Direct laser write experiments were successfully performed by using PEG-DA and/or EPOX as the monomer through a computer programmed laser diode at $405 \mathrm{~nm}$ (Thorlabs). Photosensitive formulations also containing dyes/Iod/amine-based PIS and/without metal salt $\left(\mathrm{AgNO}_{3}\right)$ were prepared in solvent-free conditions. Writing processes were all carried out under air at room temperature and the obtained 3D patterns were analyzed using a numerical optical microscope (DSX-HRSU from Olympus Corporation) [25]. 


\subsubsection{Swelling Experiments of the obtained polymers}

Single-network and interpenetrated polymer networks obtained from laser writing were immersed into deionized water for $24 \mathrm{~h}$ at room temperature to study their swelling properties. Furthermore, the wet weight $\left(\mathrm{W}_{\mathrm{t}}\right)$ and volume $\left(\mathrm{V}_{2}\right)$ of the polymers after reaching the swelling equilibrium were measured and compared with their initial wet weights $\left(\mathrm{W}_{0}\right)$ and volumes $\left(\mathrm{V}_{1}\right)$. After that, the absorbed water was removed by heating in an $40^{\circ} \mathrm{C}$ oven, and their weights and volumes were also compared with their initial values $[27,35]$.

\subsubsection{Shape-Memory Effects of Single and IPN polymers containing/without AgNPs (4D behavior)}

Swelling and dehydration have been conducted to study the reversible temporary deformation effects of the 3D single networks and the interpenetrating polymer networks obtained by using molds. In detail, the obtained 3D polymers were firstly immersed into deionized water at room temperature to observe their deformation processes; after that, the deformed polymers were dehydrated in an oven to observe their morphological change processes (recovery or even reverse deformation processes). Finally, the samples were placed at room temperature to restore their original appearances $[37,38]$.

\subsection{Antibacterial effect of IPN containing/without AgNPs.}

As AgNPs are well-known to exhibit a broad range of antibacterial activities, in this work, gram-positive bacteria Staphylococcus aureus (CIP4.83, Pasteur institute collection) and gram-negative bacteria Pseudomonas aeruginosa (strain PAO) were selected as bacterial model to investigate the antibacterial effects of the fabricated IPN polymers containing AgNPs.

Bacterial strains were stored in glycerol $15 \%$ at $-80{ }^{\circ} \mathrm{C}$. Strains were cultivated in LB medium (Lennox L: 1\% Bacto-Tryptone, 0.5\% Bacto-Yeast Extract, $1 \% \mathrm{NaCl}$, $\left.\mathrm{NaOH}\left[3 \times 10^{-3} \mathrm{M}\right]\right)$ at $37^{\circ} \mathrm{C}$. Before the contact with IPNs, an overnight culture was 
used to start a new culture until $\mathrm{OD}_{580 \mathrm{~nm}}=1$ (approximatively $10^{9}$ bacteria/ mL). 100 $\mu \mathrm{L}$ were used to inoculate either a new LB liquid medium, a LB agar Petri dish, or the PBS buffer (No growth, but adhesion possible). After one night of growth in contact with the IPN, the antibacterial effect was observed by eyes, and by counting the colonyforming unit (CFU/ml). IPNs were remove from the medium, washed gently three times with PBS and strongly vortex in PBS 10 minutes in order to detach adhered bacterial cells. CFU were again evaluated by spreading appropriates dilutions. Controls without IPN, with IPN but without AgNPs, were systematically perform in parallel.

\section{Results and Discussion}

\subsection{Photopolymerization ability of the Proposed Dyes-based PISs upon visible} light.

\subsubsection{Free radical polymerization (FRP) of PEG-DA.}

At first, the visible light source, namely the LED@405nm, was used to examine the photoinitiating ability of the dye/Iod/amine $(0.1 \mathrm{wt} \% / 1.5 \mathrm{wt} \% / 1.5 \mathrm{wt} \%)$-based threecompound PIS during the FRP of PEG-DA at room temperature. We monitored the photopolymerization kinetics of the different formulations with thicknesses of about 20 $\mu \mathrm{m}$ (thin films) and $2 \mathrm{~mm}$ (thick films) upon light irradiation for $200 \mathrm{~s}$. Polymerization profiles are shown in Figure 1. For the thin films, compared to the blank control which is only composed of the two-component Iod/amine combination, all the newly proposed dyes-based three-component PIS showed better photoinitiation efficiencies (the increased final functional groups conversions and /or the faster photopolymerization speed), which proved that the presence of the dyes was essential to improve the overall polymerization performance. In addition, under the same conditions, for thick samples with the thickness of about $2 \mathrm{~mm}$, except for the three-component PIS based on dyes 6 , 8 and 9, all the other PISs showed better photoinitiation abilities than the blank control (conversion rate $\geq 90 \%$ of the experimental group vs. $87 \%$ of the blank group).

More interestingly, under a LED@405nm irradiation, whether for thin films or thick films, the final conversion rates achieved by the PIS based on dyes 1, 2, 3, 4, 5, 9 and 11 were greater than $90 \%$, which can be attributable to their higher molar extinction coefficients. 
(a)

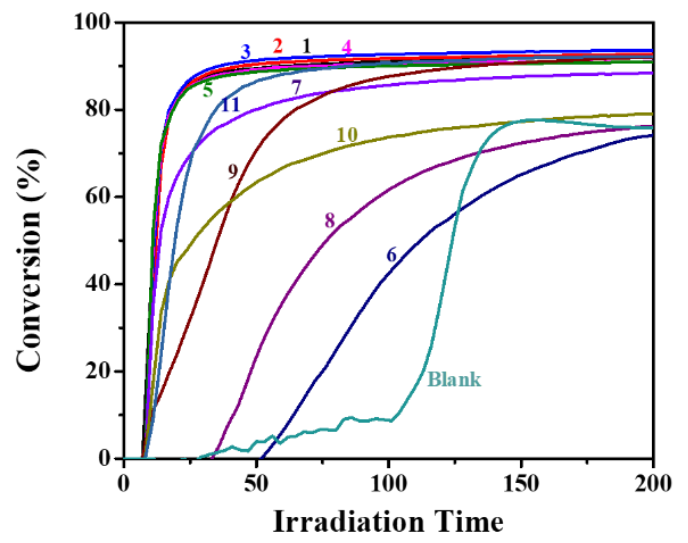

(b)

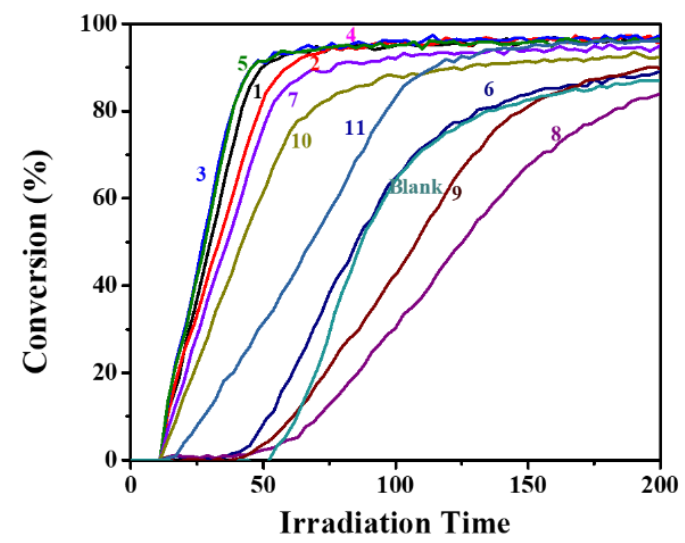

Figure 1. Photopolymerization profiles of PEG-DA (conversion of $\mathrm{C}=\mathrm{C}$ bonds $v s$ irradiation time) initiated by dyes/Iod/amine $(0.1 \mathrm{wt} \% / 1.5 \mathrm{wt} \% / 1.5 \mathrm{wt} \%$, w/w/w) in laminate upon irradiation with LED@405nm $\left(\mathrm{I}_{0}=110 \mathrm{~mW} . \mathrm{cm}^{-2}\right)$ with the thickness of about (a) $20 \mu \mathrm{m}$ and (b) $2 \mathrm{~mm}$, the irradiation starts at $\mathrm{t}=10 \mathrm{~s}$.

\subsubsection{Cationic polymerization (CP) of EPOX.}

At the same time, under the same conditions, photoinitiation abilities of the threecomponent PIS during the cationic photopolymerization of EPOX has also been studied. As shown in Figure 2, it is obvious that only using Iod and amine as the twocompound PIS cannot convert the EPOX resin from liquid into solid, even after $200 \mathrm{~s}$ of light irradiation, while in the case of thin films initiated by the proposed dyes-based three-component PIS, deep curing has been observed for all of the formulations. However, for thick films with a thickness of about $2 \mathrm{~mm}$, only the three-component PISs based on dyes 1, 2, 3, 4 and 5 could initiate the deep curing of EPOX formulations. All these results highlight the crucial role of the three-component systems for the overall performance.

(a)

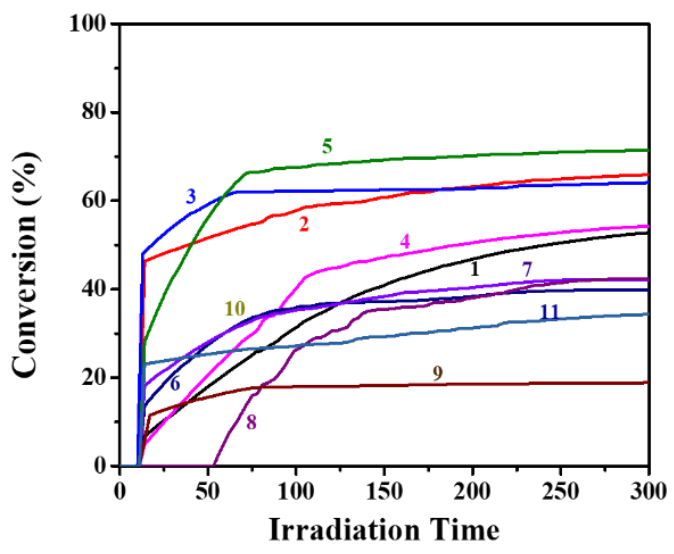

(b)

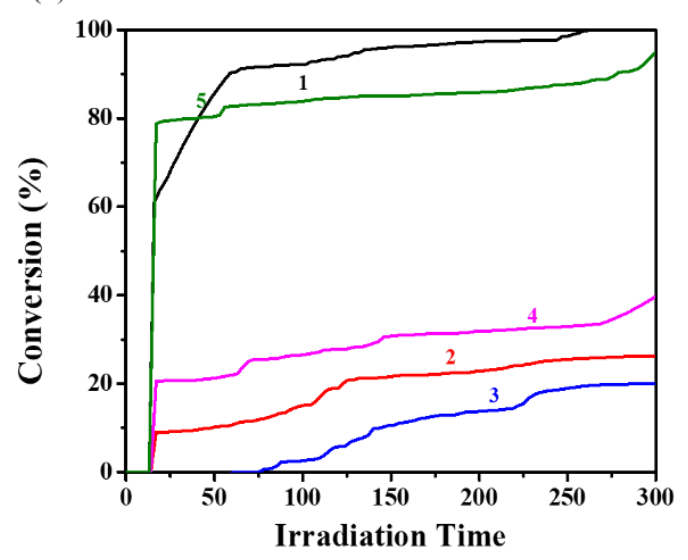


Figure 2. Photopolymerization profiles of EPOX (conversion of epoxy functions $v s$ irradiation time) initiated by dyes/Iod/amine $(0.1 \mathrm{wt} \% / 1.5 \mathrm{wt} \% / 1.5 \mathrm{wt} \%$, w/w/w) under air upon irradiation with a LED@405nm $\left(\mathrm{I}_{0}=110 \mathrm{~mW} . \mathrm{cm}^{-2}\right)$ with the thickness about (a) $20 \mu \mathrm{m}$ and (b) $2 \mathrm{~mm}$, the irradiation starts at $\mathrm{t}=10 \mathrm{~s}$.

\begin{tabular}{|c|c|c|c|c|}
\hline \multicolumn{5}{|c|}{ Final acrylate function conversions (FC) initiated by dye/Iod/amine } \\
\hline \multirow[b]{2}{*}{ Dyes } & \multicolumn{2}{|c|}{ Thin files $\sim 20 \mu \mathrm{m}$} & \multicolumn{2}{|c|}{ Thin files $\sim 2 \mathrm{~mm}$} \\
\hline & EPOX $(\%)$ & PEG-DA $(\%)$ & EPOX $(\%)$ & PEG-DA $(\%)$ \\
\hline 1 & 53 & 92 & 99 & 96 \\
\hline 2 & 66 & 93 & 26 & 95 \\
\hline 3 & 64 & 94 & 20 & 98 \\
\hline 4 & 54 & 91 & 40 & 97 \\
\hline 5 & 71 & 91 & 95 & 97 \\
\hline 6 & 40 & 75 & - & 90 \\
\hline 7 & 42 & 88 & - & 95 \\
\hline 8 & 42 & 76 & - & 84 \\
\hline 9 & 20 & 92 & - & 90 \\
\hline 10 & 34 & 80 & - & 92 \\
\hline 11 & 35 & 92 & - & 96 \\
\hline Blank & - & 75.8 & - & 87 \\
\hline
\end{tabular}

Table 1. Summary of the final function conversions for PEG-DA and EPOX monomers while using dyes/Iod/amine-based PISs with a thickness about $20 \mu \mathrm{m}$ and $2 \mathrm{~mm}$ upon irradiation with a LED@405nm. The blank corresponds to the Iod/amine initiating system without dyes.

\subsubsection{The fabrication of IPN polymers through different ratio of PEG-DA and EPOX.}

Based on the abovementioned results, dyes 1-5/Iod/amine based three-component PIS proved to be the best candidates as all of them could not only initiate the 
photopolymerization of PEG-DA, but also could promote the deep curing of EPOX. In addition, a variety of studies have shown that the interpenetrating polymer networks have their unique structures and properties compared with the single polymer networks, which broadening their applications $[8,10]$. In this work, we further studied the initiating ability of the newly proposed dyes-based PISs for PEG-DA/EPOX mixed monomers. Three ratios of resins based on mixed monomers were prepared (in detail, PEG-DA: EPOX = 3:7, 5:5, 7:3). Their polymerization curves are shown in Figure S1 and the final function conversions (acrylate and epoxide) are summarized in Table 2. All of them were determined for a 70/ 30 ratio of PEG-DA/EPOX monomers which was determined as the best composition in terms of polymerization efficiencies for the two functionalities (acrylates and epoxides) [39].

\begin{tabular}{|c|c|c|c|c|c|c|c|c|c|c|}
\hline \multirow[t]{2}{*}{ dye } & \multicolumn{5}{|c|}{ Thin (PEG-DA/EPOX) } & \multicolumn{5}{|c|}{ Thick (PEG-DA/EPOX) } \\
\hline & 100:0 & $70: 30$ & $50: 50$ & $30: 70$ & $0: 100$ & 100:0 & $70: 30$ & $50: 50$ & $30: 70$ & $0: 100$ \\
\hline 1 & $92 \%$ & $94 \% / 57 \%$ & $34 \% / 23 \%$ & $25 \% / 8 \%$ & $38 \%$ & $96 \%$ & $95 \% / 46 \%$ & $94 \% / 27 \%$ & $96 \% / 45 \%$ & $97 \%$ \\
\hline 2 & $93 \%$ & $82 \% / 53 \%$ & $31 \% / 13 \%$ & $76 \% / 62 \%$ & $19 \%$ & $96 \%$ & $95 \% / 39 \%$ & $94 \% / 26 \%$ & $85 \% / 46 \%$ & $23 \%$ \\
\hline 3 & $94 \%$ & $82 \% / 48 \%$ & $96 \% / 44 \%$ & $20 \% / 50 \%$ & $63 \%$ & $98 \%$ & $94 \% / 41 \%$ & $64 \% / 44 \%$ & $93 \% / 63 \%$ & $14 \%$ \\
\hline 4 & $91 \%$ & $76 \% / 24 \%$ & $85 \% / 67 \%$ & $78 \% 56 \%$ & $34 \%$ & $97 \%$ & $91 \% / 55 \%$ & $98 \% / 67 \%$ & $83 \% / 69 \%$ & $32 \%$ \\
\hline 5 & $91 \%$ & $80 \% / 44 \%$ & $70 \% / 27 \%$ & $29 \% / 26 \%$ & $31 \%$ & $97 \%$ & $93 \% / 41 \%$ & $94 \% / 27 \%$ & $95 \% / 51 \%$ & $86 \%$ \\
\hline
\end{tabular}

Table 2. Summary of the final function conversions for PEG-DA and EPOX monomers while using dyes/Iod/amine-based PISs for different ratio of PEG-DA/EPOX mixed monomers with the thickness about $20 \mu \mathrm{m}$ and $2 \mathrm{~mm}$ upon irradiation with a LED@405nm.

\subsection{The involved chemical mechanisms}


Based on the photoinitiation ability of the eleven different dyes based PIS, dyes 15 were selected as the best candidates for following researches devoted to investigate the photo-physicochemical properties and examine the involved chemical mechanisms.

\subsubsection{UV-vis Absorption Properties of the Proposed Dyes.}

Firstly, the UV-visible absorption properties of the eleven dyes have been examined in acetonitrile and their absorption spectra are shown in Figure 3. Except for dyes 6,8 and 9 that only exhibited an absorption peak in the near UV region, all the other dyes showed two or three absorption peaks in the UV and visible region, in which the maximum absorption peaks appeared in the near visible region ( $\geq 390 \mathrm{~nm})$. These absorption maxima perfectly overlap with the emission wavelength of the visible light sources (LED@405nm). Notably, their absorption wavelengths $\left(\lambda_{\max }\right)$, their molar extinction coefficients at maximum absorption wavelengths $\left(\varepsilon_{\max }\right)$ and at the emission wavelength of the visible light source LED@405 nm ( $\varepsilon @ 405 \mathrm{~nm})$ are summarized in Table 1. More interestingly, the strongest absorption peaks of the selected five dyes all appeared between $380 \sim 400 \mathrm{~nm}$, meanwhile, all of them also showed higher molar extinction coefficients $\left(\varepsilon_{\max }\right)$ at $405 \mathrm{~nm}$ (such as $20140 \mathrm{M}^{-1} \mathrm{~cm}^{-1}, 18390 \mathrm{M}^{-1} \mathrm{~cm}^{-1}, 9350$ $\mathrm{M}^{-1} \mathrm{~cm}^{-1}, 12545 \mathrm{M}^{-1} \mathrm{~cm}^{-1}$ and $12130 \mathrm{M}^{-1} \mathrm{~cm}^{-1}$ for these five dyes, respectively), which greatly matched with their excellent photoinitiation abilities. Remarkably, introduction of electron-donating groups caused a red-shift of the absorption spectra.

(a)

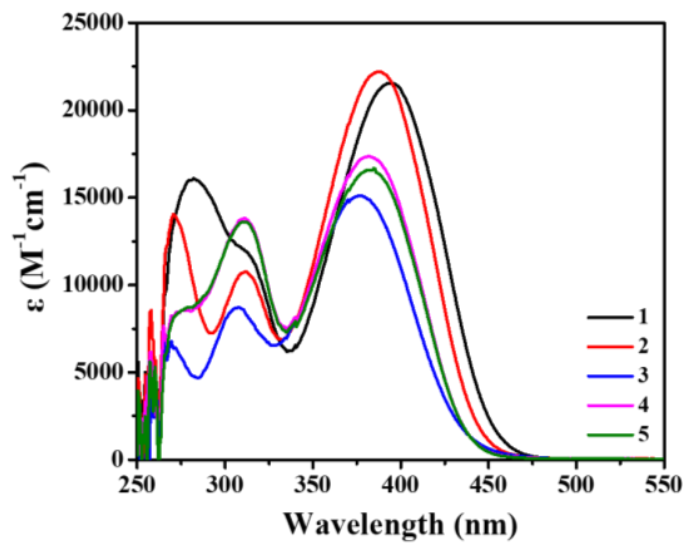

(b)

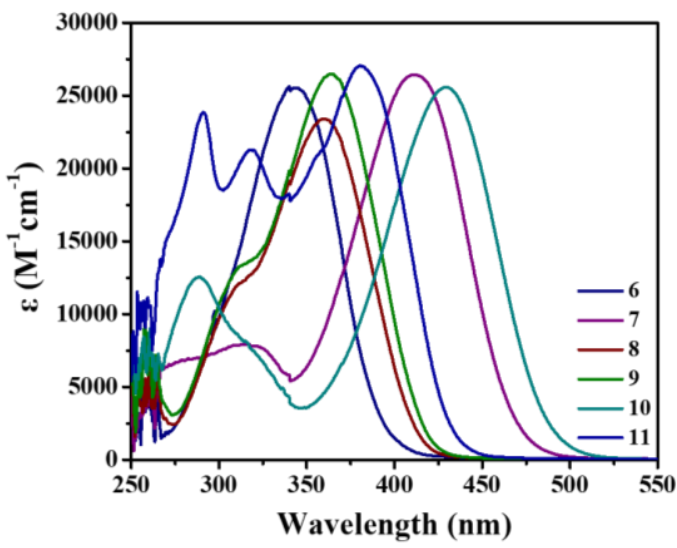

Figure 3. Light absorption properties of (a) dyes $1 \sim 5$ and (b) dyes $6 \sim 11$ in acetonitrile. 


\begin{tabular}{|c|c|c|c|}
\hline DYEs & $\lambda_{\max (\mathrm{nm})}$ & $\varepsilon_{\max }\left(M^{-1} \mathrm{~cm}^{-1}\right)$ & $\varepsilon_{@ 405 \mathrm{~nm}}\left(M^{-1} \mathrm{~cm}^{-1}\right)$ \\
\hline 1 & $\begin{array}{l}395 \\
282\end{array}$ & $\begin{array}{l}21560 \\
16100\end{array}$ & 20140 \\
\hline 2 & $\begin{array}{l}388 \\
312 \\
270\end{array}$ & $\begin{array}{l}22200 \\
10770 \\
14040\end{array}$ & 18390 \\
\hline 3 & $\begin{array}{l}378 \\
307\end{array}$ & $\begin{array}{r}15090 \\
8720\end{array}$ & 9350 \\
\hline 4 & $\begin{array}{l}382 \\
311\end{array}$ & $\begin{array}{l}17360 \\
13820\end{array}$ & 12545 \\
\hline 5 & $\begin{array}{l}385 \\
312 \\
\end{array}$ & $\begin{array}{l}16660 \\
13610\end{array}$ & 12130 \\
\hline 6 & 340 & 25620 & 970 \\
\hline 7 & $\begin{array}{l}411 \\
316\end{array}$ & $\begin{array}{r}26440 \\
7990\end{array}$ & 26010 \\
\hline 8 & 360 & 23400 & 4290 \\
\hline 9 & 364 & 26500 & 6760 \\
\hline 10 & $\begin{array}{l}430 \\
289\end{array}$ & $\begin{array}{l}25560 \\
12570\end{array}$ & 19500 \\
\hline 11 & $\begin{array}{l}380 \\
317 \\
291\end{array}$ & $\begin{array}{ll}27 & 030 \\
21 & 270 \\
23 & 850\end{array}$ & 17480 \\
\hline
\end{tabular}

Table 3. Light absorption properties of dyes 1-11 in acetonitrile: maximum absorption wavelengths $\lambda_{\max }$; molar extinction coefficients at $\lambda_{\max }\left(\varepsilon_{\max }\right)$ and the emission wavelength of the LED@405 nm (E@405nm).

\subsubsection{Steady state photolysis.}

After studying the UV-visible absorption properties of the different dyes, interactions between the proposed dyes and Iod or amines have been examined through the steady-state photolysis measurements of one compound-, two compounds- and three compounds-systems based on dye, dye/Iod, dye/amine and dye/Iod/amine in acetonitrile. To be comparable to the conditions used during photopolymerization, photolysis experiments were carried out using a visible light LED@405 nm. As shown in Figure 4, S1 and S2, obvious photolyses have been observed for all samples within 1 min of light irradiation so that the absorbance decreased significantly. Furthermore, 
according to their photolyses, consumptions of the dyes have also been calculated to intuitively measure the efficiency of the reactions between dyes with Iod/amines. For dye 1 (as shown in Figure 4), the three-component PIS achieved the maximum consumption of dyes, which was about $80 \%$ (see Figure 4(d)); Conversely, the twocomponent dye/Iod based PIS only achieved about 50\% (see Figure 4(b)) consumption; Finally, for the other two-component dye/amine PIS and the dyes alone, only $10 \%$ (see Figure $4(\mathrm{a}$ and $\mathrm{b})$ ) consumption could be achieved. Interestingly, for dyes 2 and 3 (see Figure S1), the maximum consumption was achieved with the dye/Iod based twocomponent PIS, which could reach about 90\%; the second one was the dyes/Iod/aminebased three-component PIS, that was about 40\%; the dyes/amines and dyes alone based PIS also achieved the lowest consumption ( $20 \%)$. The above results may be due to the introduction of electron-donating groups into the structures of dyes 2 and 3. Indeed, the internal electron transfer in the molecule will compete with the electron transfer between the dye and the amine, thereby slowing down the reactions between the dyes and Iod as well as the amine, which decrease the dyes consumption [40,41]. More interestingly, photolysis processes of dyes 4 and 5- based systems were completed within $15 \mathrm{~s}$ (see Figure S2), which is faster than that observed for the other three dyes; furthermore, dyes consumptions achieved by these four different systems were similar and were about $40 \%$. In addition, the absorption peak around $310 \mathrm{~nm}$ also showed a blue shift upon light irradiation for the dyes 4 and 5-based systems. 
(a)

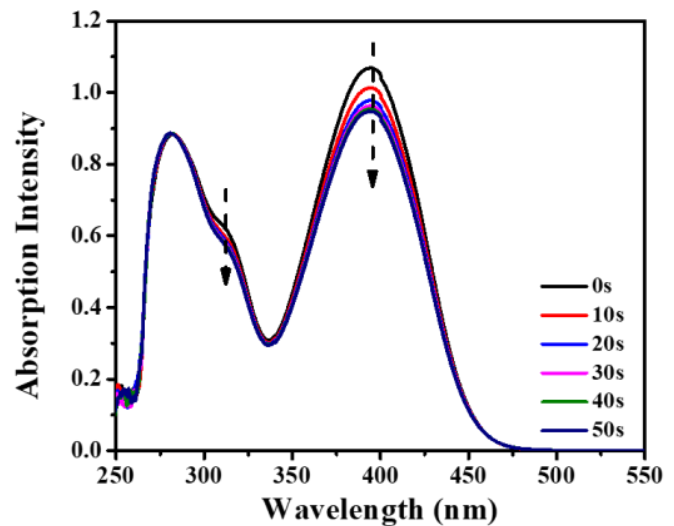

(c)

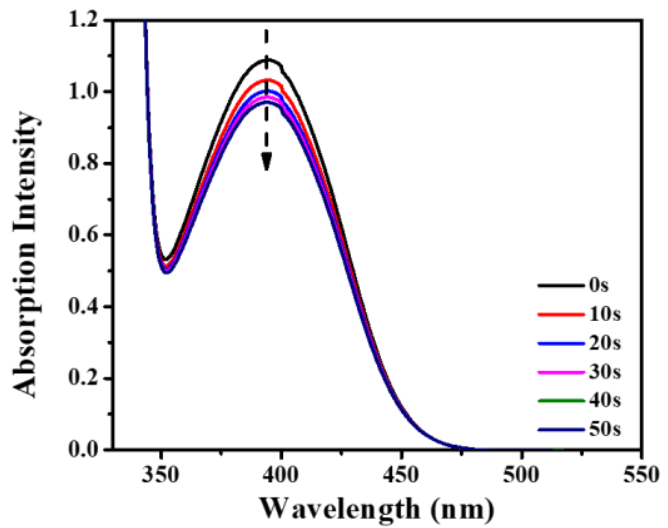

(b)

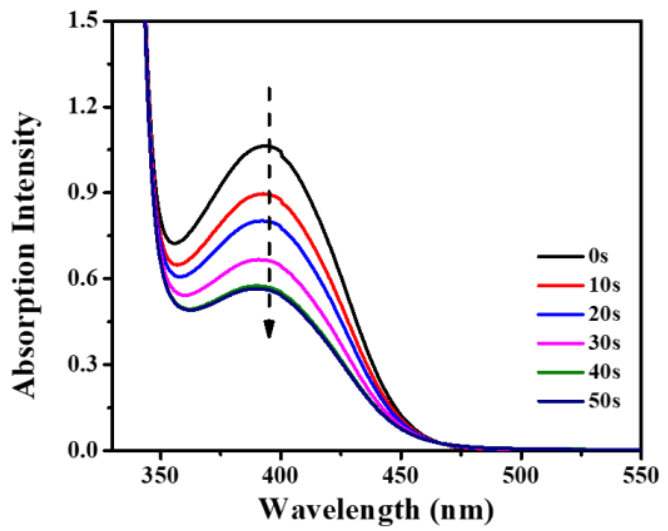

(d)

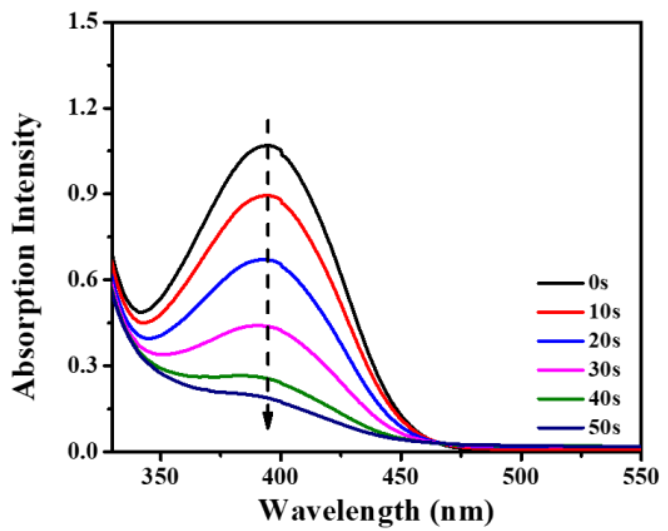

Figure 4. Steady state photolysis of (a) dye 1; (b) dye 1/Iod; (c)dye 1/amine and (d) dye 1/Iod/amine-based formulations upon exposure to LED@405nm under air in acetonitrile. 


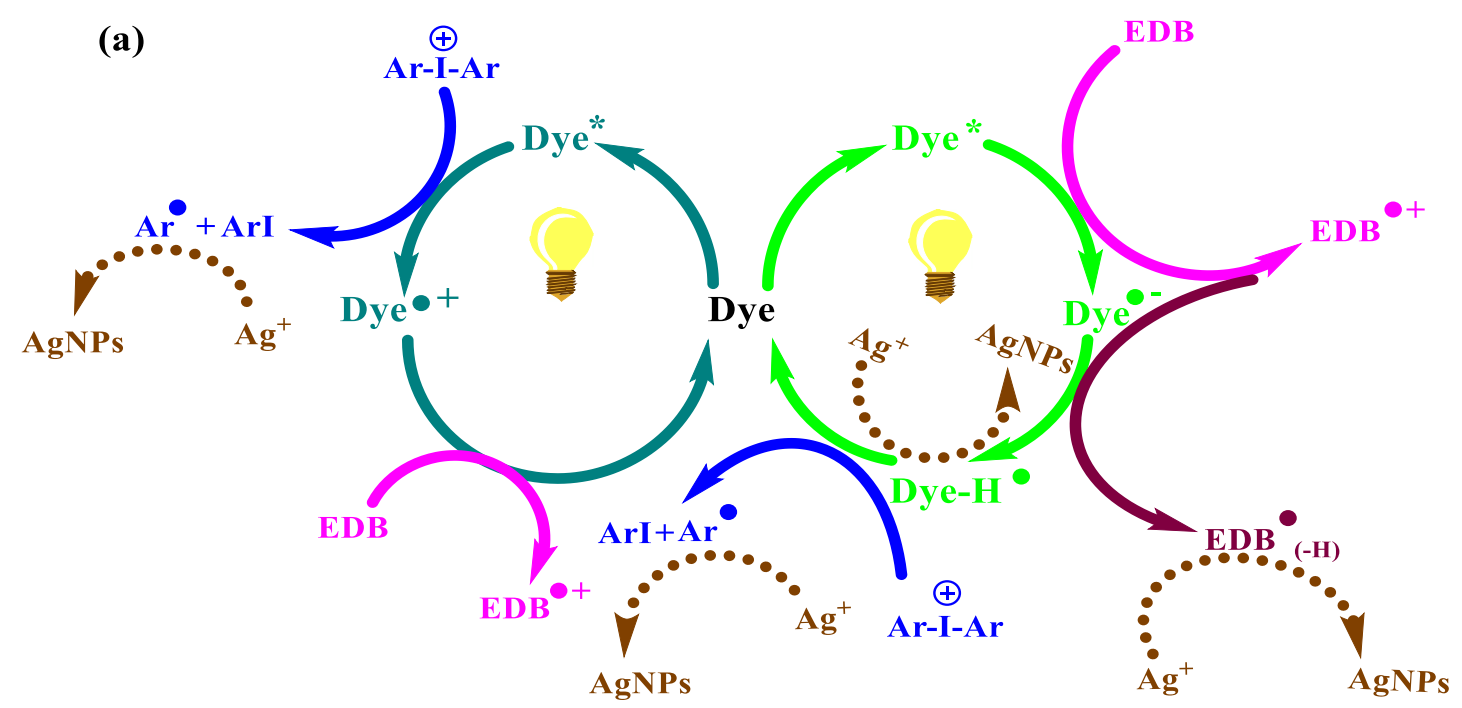

(b)

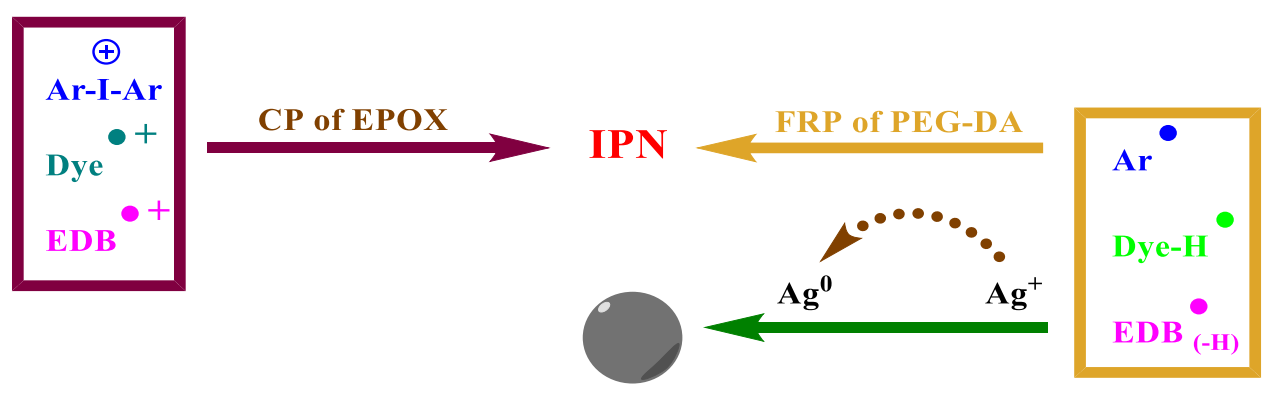

$\operatorname{AgNPs}$

Scheme 3. (a) Proposed photoinitiation step mechanisms for the three-component dyes/Iod/amine initiating systems; (b) Photoinitiation mechanism for the preparation of IPN with metallic salts under air atmosphere.

\subsubsection{Fluorescence quenching experiments.}

After that, the electron transfer efficiency between dyes 1-5 and Iod/EDB have also been studied through fluorescence quenching experiments. For dye 1, a significant decrease of the emission intensity has been observed with the addition of Iod, while with the addition of the amine, intensity of the emission peak gradually increased, and a blue shift was observed. Furthermore, as shown in Figure S5 (c-f), the gradual addition of Iod/EDB could decrease the emission intensities of dyes 2 and 3 based formulations; more interestingly, interaction between Iod and these two dyes were much stronger than that between dyes and amine. A blue-shift can also be observed upon addition of EDB. Remarkably, Figure 5 clearly presents the fluorescence 
quenching of dyes 4 and 5 in the presence of Iod/EDB, whose fluorescence emission intensity greatly decreased, which proves that Iod and the amine can act as good quenchers during the interaction between the dye with Iod/amine in the excited state. A linear curve can be fitted from the fluorescence quenching process between dyes 4 and 5 with Iod/amine.

In addition, the Stern-Volmer coefficients $\left(\mathrm{K}_{\mathrm{SV}}\right.$, slope of the curve, summarized in Table 3$)$ and the electron transfer quantum yields ( $\varphi_{\mathrm{et}}$, summaried in Table 3 ) of the electron transfer reaction between these dyes and Iod or amine were calculated by using their fluorescence quenching curve, and all of them are positively correlated with the efficiency of electron transfer. 
(a)

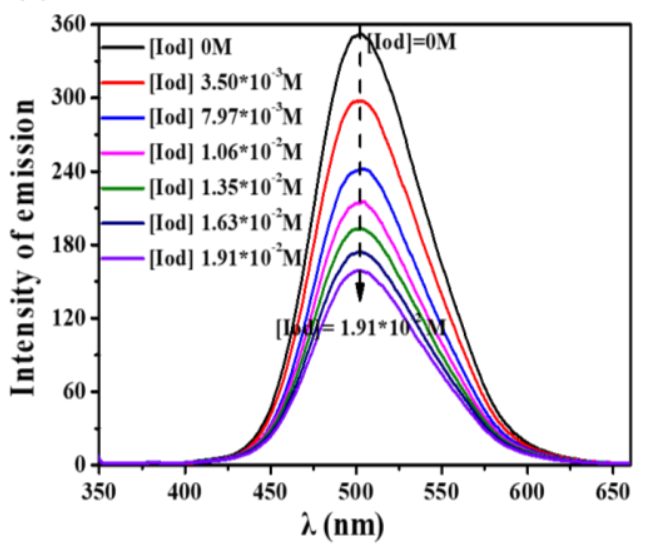

(c)

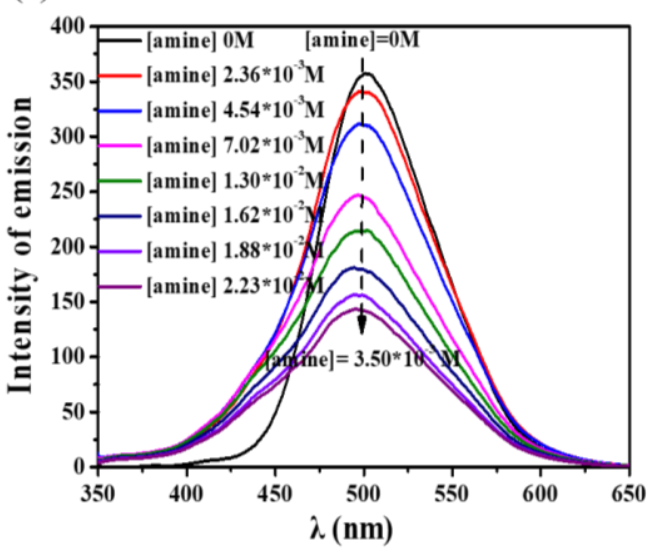

(e)

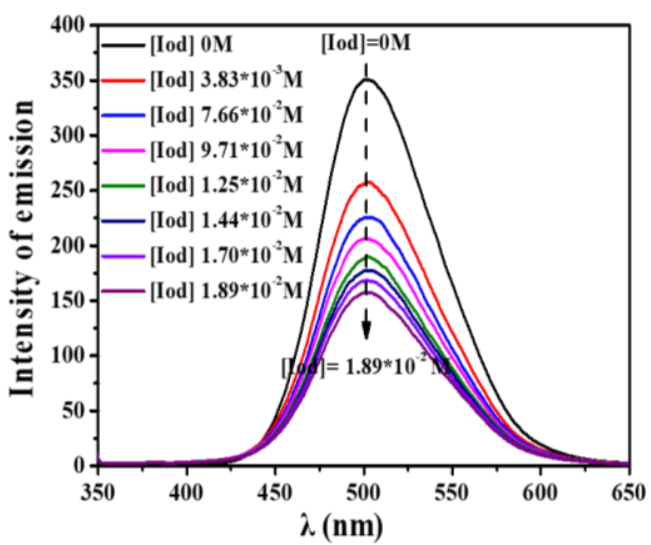

(g)

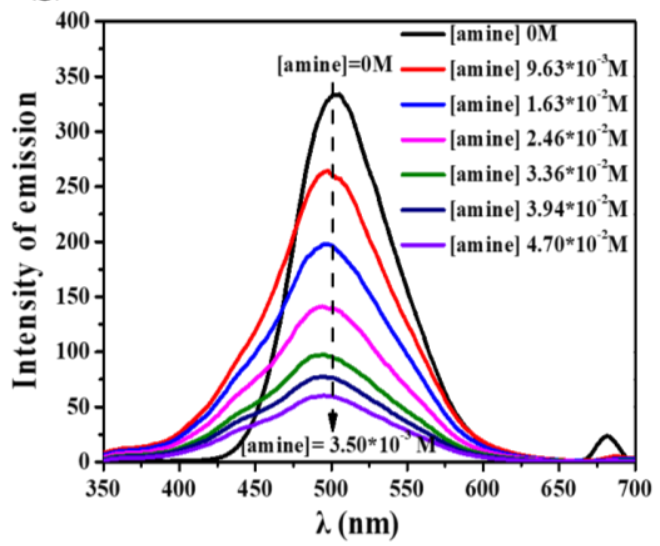

(b)

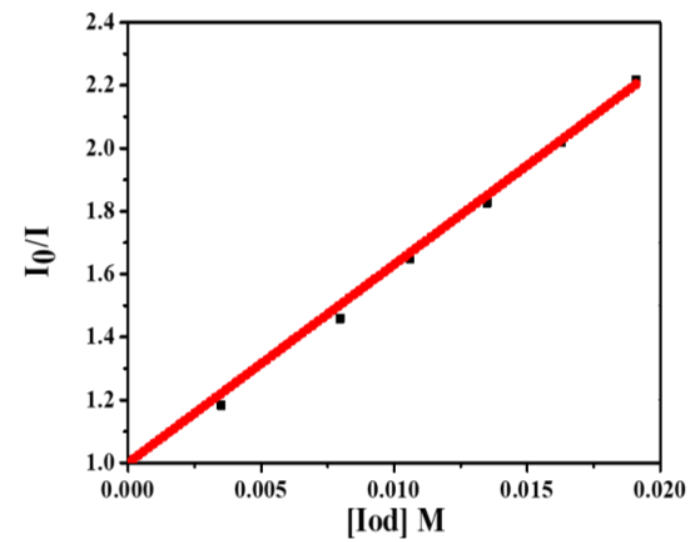

(d)

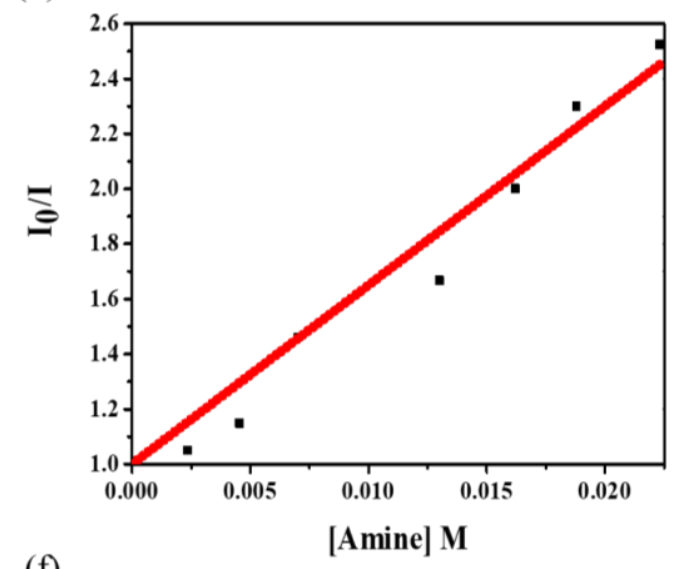

(f)

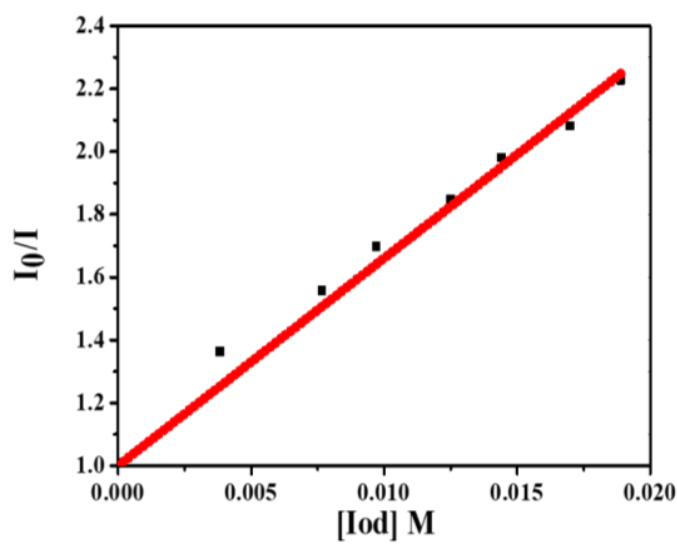

(h)

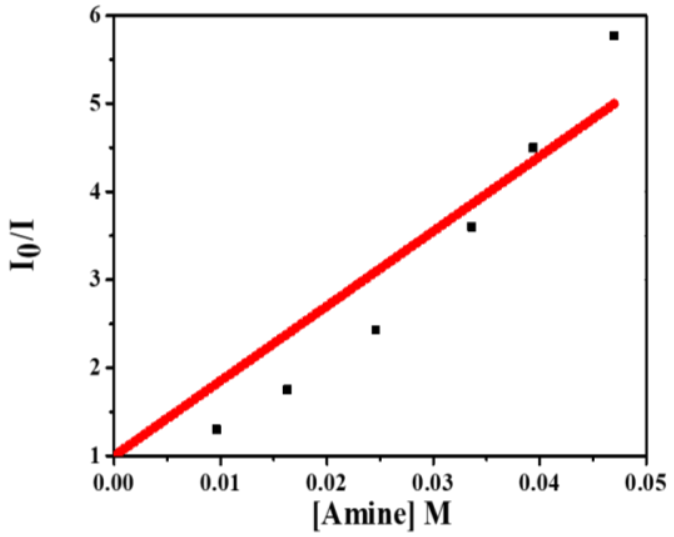


Figure 5. (a) Fluorescence quenching of dye 4 by Iod (speedcure 938); (b) Stern-Volmer treatment for the dye 4/Iod fluorescence quenching; (C) Fluorescence quenching of dye 4 by amine (speedcure EDB); (D) Stern-Volmer treatment for the dye 4/EDB fluorescence quenching; (E) Fluorescence quenching of dye 5 by Iod (speedcure 938); (F) Stern-Volmer treatment for the dye 5/Iod fluorescence quenching; (G) Fluorescence quenching of dye 5 by amine (speedcure EDB); (H) Stern-Volmer treatment for the dye 5/EDB fluorescence quenching.

\subsubsection{Feasibility of the interactions between dyes and Iod/amines}

Based on the above results concerning their UV/visible absorption properties and fluorescence characteristics, their first singlet excited state energy ( $E_{S 1}$, see Table 4) can be obtained from the crossing point of their standardized visible light absorption and fluorescence spectra. Furthermore, the oxidation potential and reduction potential of these dyes were detected by cyclic voltammetry and determined through the position of their oxidation and reduction peaks (see Table 4). As shown in Figure 5, a clear oxidation peak can be observed in their positive potential range, among which a weak oxidation peak can also be detected for dyes 1 and 2 . However, in the negative potential range, an obvious reduction peak can also be observed for all dyes, in addition, dyes 25 also possess a weak reduction peak. Finally, according to the above-mentioned parameters summarizing in Table 4 , the free energy change $\left(\Delta \mathrm{G}_{\mathrm{Iod}}\right.$ or $\left.\Delta \mathrm{G}_{\mathrm{EDB}}\right)$ of the electron transfer reactions between dye/Iod (or dye/amine) were calculated using equations (3), and all of them were less than 0 , indicating the theoretical/practical feasibility of the interactions between the dyes and Iod/amines, which further proved that reactions ( as shown in Scheme 3 ) can occur and can generate the corresponding free radicals and cations to initiate the polymerization of mixed monomers under visible light.

\begin{tabular}{lccccc}
\hline & Dye 1 & Dye 2 & Dye 3 & Dye 4 & Dye 5 \\
$\mathbf{K}_{\text {sv }}{ }^{\text {Iod }}\left(\mathbf{M}^{-1}\right)$ & 30 & 29 & 51 & 36 & 66 \\
\hline
\end{tabular}




\begin{tabular}{|c|c|c|c|c|c|}
\hline$K_{s v}{ }^{E D B}\left(M^{-1}\right)$ & - & 6 & - & 68 & 63 \\
\hline$\Phi_{\text {et }}^{\text {Iod }}$ & 0.48 & 0.48 & 0.61 & 0.53 & 0.67 \\
\hline$\Phi_{\text {et }}^{\text {EDB }}$ & - & 0.16 & - & 0.86 & 0.85 \\
\hline $\mathbf{E}_{\mathrm{s} 1}(\mathrm{eV})$ & 2.7 & 2.76 & 2.80 & 2.81 & 2.82 \\
\hline \multicolumn{6}{|l|}{$\mathbf{E}_{\mathrm{T} 1}(\mathrm{eV})$} \\
\hline $\mathbf{E}^{\mathbf{o x}}(\mathrm{eV})$ & 1.13 & 1.17 & 1.1 & 1.1 & 1.08 \\
\hline $\mathbf{E}^{\text {red }}(\mathrm{eV})$ & -1.49 & -1.57 & -1.62 & -1.64 & -1.69 \\
\hline$\Delta \mathbf{G}_{\mathrm{S} 1}{ }^{\text {Iod }}$ & -0.87 & -0.89 & -1 & -1.01 & -1.04 \\
\hline$\Delta \mathbf{G}_{\mathrm{S} 1}{ }^{\mathrm{EDB}}$ & -0.21 & -0.19 & -0.18 & -0.17 & -0.13 \\
\hline \multicolumn{6}{|l|}{$\Delta \mathbf{G}_{\mathrm{T} 1}{ }^{\text {Iod }}$} \\
\hline$\Delta \mathbf{G}_{\mathrm{T} 1}{ }^{\text {EDB }}$ & & & & & \\
\hline
\end{tabular}

Table 4. Summary of the relevant parameters of the fluorescence properties of dyes 15 , namely the interaction constant $\left(\mathrm{K}_{\mathrm{sv}}\right)$ and electron transfer quantum yield $\left(\Phi_{\mathrm{et}}\right)$ of the dye-Iod and dye-EDB systems; singlet excited state energy $\left(E_{S 1}\right)$ and oxidation/reduction potential $\left(\mathrm{E}_{\mathrm{ox}} / \mathrm{E}_{\mathrm{red}}\right)$ of dyes 1-5 as well as the free energy change of the electron transfer reaction between dye and $\operatorname{Iod} / \mathrm{EDB}\left(\Delta \mathrm{G}_{\mathrm{S} 1}\right)$. 
(a)

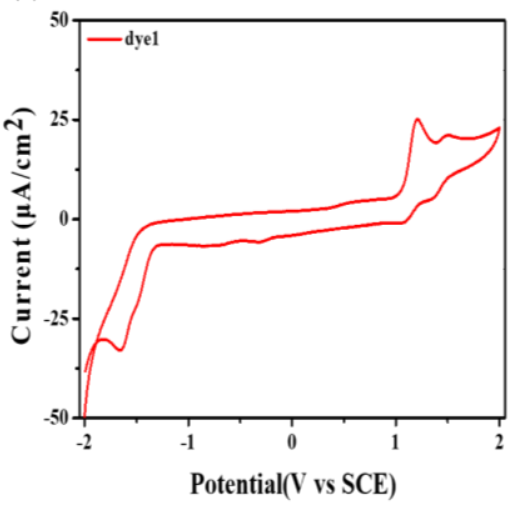

(d)

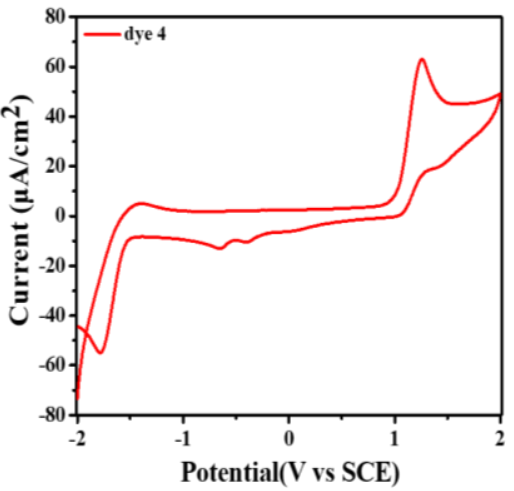

(b)

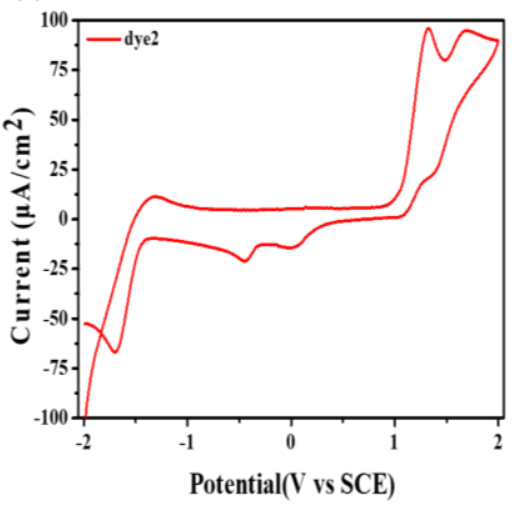

(e) (c)

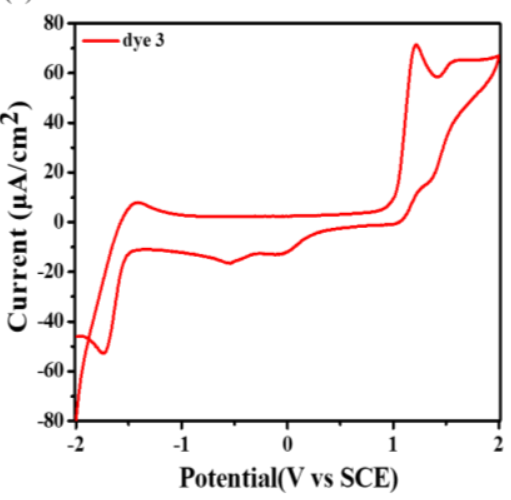

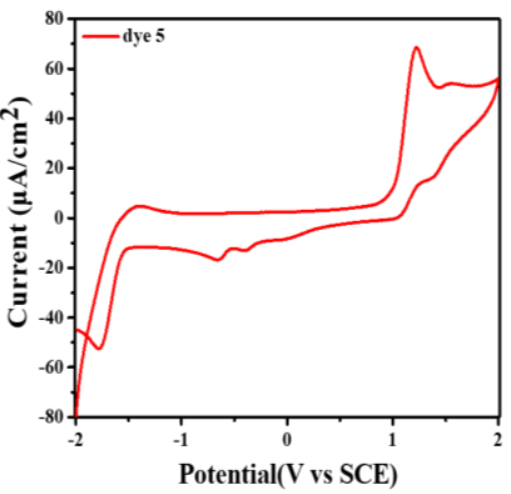

Figure 6. Cyclic voltammograms of dyes 1-5 in acetonitrile.

\subsubsection{ESR-spin trapping experiments for dye 5 with Iod/EDB formulations}

It has been proved that the proposed redox electron transfer reaction can occur, as demonstrated with the above results. Furthermore, the generated radicals have also been detected by ESR-spin trapping experiments upon irradiation with sunlight of dye 5-Iod and dye 5-amine solutions. As shown in Figure S5, aryl radicals were trapped in the dye 5-Iod solutions, characterized by the hyperfine coupling constants of nitrogen and hydrogen: $\mathrm{a}_{\mathrm{N}}=14.4 \mathrm{G}$ and $\mathrm{a}_{\mathrm{H}}=2.1 \mathrm{G}$, which are in full agreement with the literature [36]. Meanwhile, under sunlight irradiation, aminoalkyl radicals $\left(\mathrm{a}_{\mathrm{N}}=14.4 \mathrm{G}\right.$ and $\mathrm{a}_{\mathrm{H}}=$ $2.5 \mathrm{G}, 90 \%,[33])$ and a few of other free radicals such as PBNox were captured from the dye 5-amine solution. All of them also proved that the series of electron transfer reactions proposed in Scheme 3 can occur during the photopolymerization process. 


\subsubsection{AgNPs generated in DMF solution and IPN-polymers.}

According to our previous studies, it has been proved that the redox reactions performed between some dyes-based PIs and additives Iod/amine under visible light irradiation can generate free radicals and cations [25], which can be used to initiate the deep curing of monomers. Meanwhile they can also reduce the metal salts into metal nanoparticles. In this work, DMF solutions containing three-component PIS (dye/Iod/EDB) and silver nitrate were irradiated upon visible light and the absorption spectra were collected at different times. As shown in Figure 6, the characteristic absorption peak of the dye at $390 \mathrm{~nm}$ gradually decreased upon visible light irradiation, indicating that the dye interacted with Iod/EDB. At the same time, a new absorption peak appeared at $430 \mathrm{~nm}$ and its absorbance gradually increased, which indicated that the $\mathrm{Ag}^{+}$cations were reduced into AgNPs $[45,46]$. In addition, the solution changed from light yellow to yellow-brown during the light irradiation process, and a yellowbrown silver nanoparticle coating was observed on the side of the cuvette. Furthermore, nanoparticles produced in DMF solutions were measured by TEM and regular spheres with a size about 3-5 $\mathrm{nm}$ were determined.

Subsequently, in order to prepare the different IPNs containing AgNPs, visible light LED@405nm was used to initiate the deep curing of the formulation based on PEG-DA/EPOX (70\%/30\%) and containing $\mathrm{AgNO}_{3}(5 \mathrm{wt} \%)$ and dye/Iod/EDB $(0.1 \mathrm{wt} \% / 1.5 \mathrm{wt} \% / 1.5 \mathrm{wt} \%)$. At the same time, the polymerization curves of the different formulations were followed by RT-FTIR. As shown in Figure 6c-f, compared to the photopolymerization of mixed monomers that do not contain $\mathrm{AgNO}_{3}$, for the thin samples with the thickness about $0.1 \mu \mathrm{m}$, presence of $\mathrm{Ag}^{+}$promoted the cationic polymerization of EPOX and weaken the free radical polymerization efficiency of PEG-DA. In addition, for the polymerization of thick samples (thickness about $2 \mathrm{~mm}$ ), presence of $\mathrm{Ag}^{+}$also improved the cationic polymerization efficiency of EPOX, while for the free radical polymerization of PEG-DA, even though their final conversions didn't changed, but the speed of the reactions (that is, the slope of the polymerization curve) have decreased. Furthermore, color of IPNs obtained after deep curing was obviously darkened and turned into dark brown. Remarkablely, AgNPs obtained from the deep-curing process of IPN-polymers were aggregated into irregular spherical 
shapes, as observed by SEM. Particle sizes at the upper surface of the polymer network were more than $100 \mathrm{~nm}$, while the size of AgNPs at the bottom of the sample were just about 30 60 nm, which is significantly larger than that produced in DMF solutions (see Figure S5). As more nanoparticles are formed at the surface of the sample due to the influence of the light penetrating ability, the particle size of AgNPs on the upper surface is larger than that at the bottom surface.

(a)

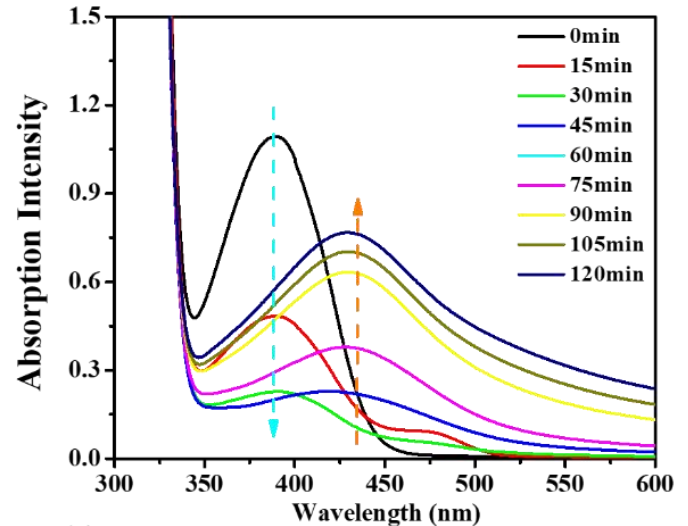

(c)

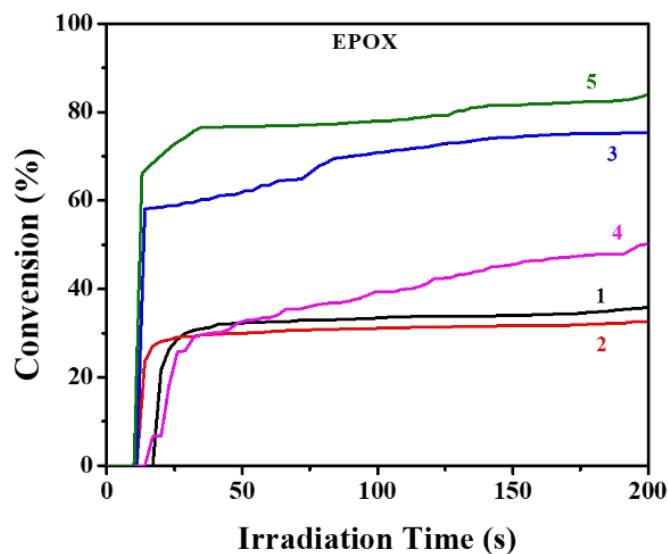

(e)

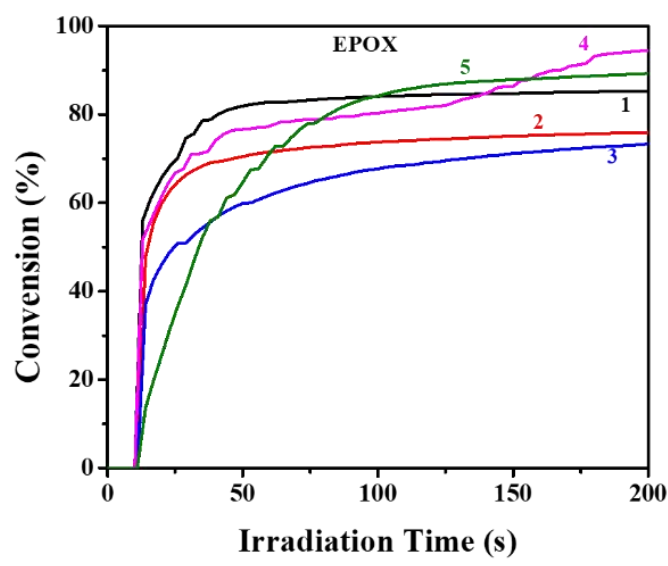

(b)

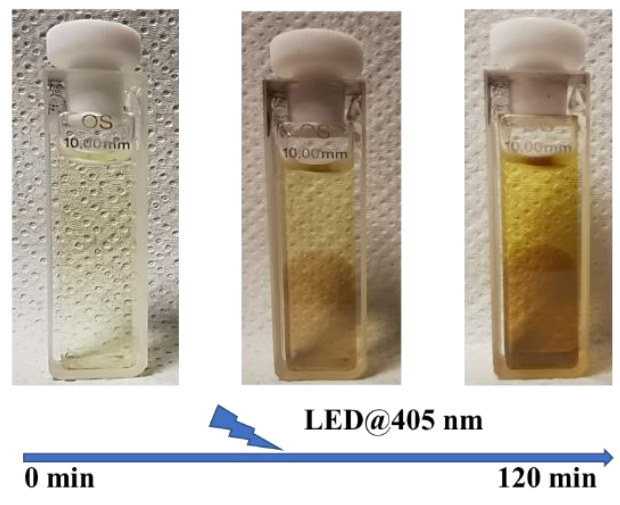

(d)

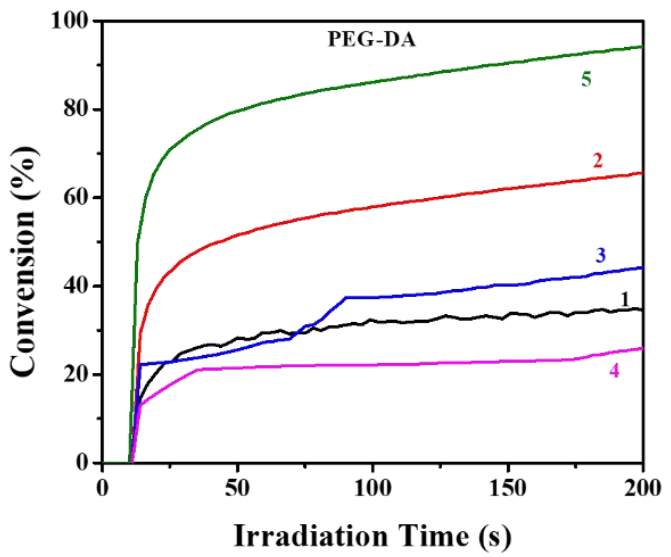

(f)

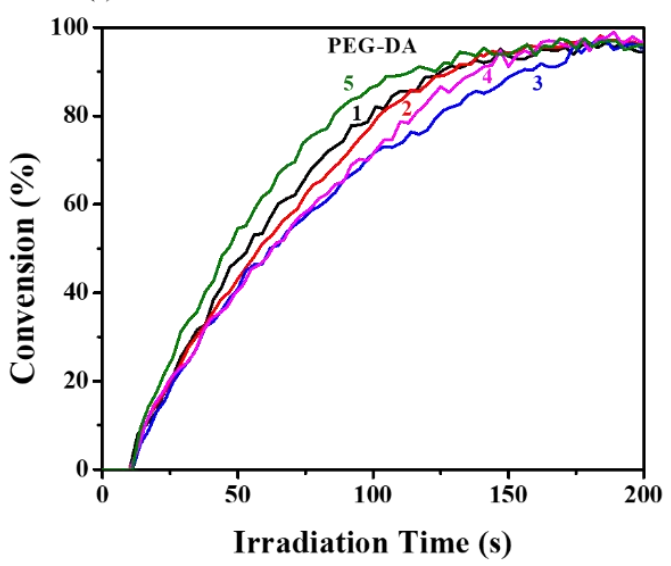

Figure 7. (a) UV-visible absorption spectra of DMF solutions containing dye 5/Iod/EDB (0.001 wt \%/0.015\%/0.015\%) and $\mathrm{AgNO}_{3}(5 \mathrm{wt} \%)$ in air atmosphere during 
120 min visible light irradiation process; (b) the changes of the color of solution after $120 \mathrm{~min}$ visible light irradiation; photopolymerization profiles of IPN (70\%PEG$\mathrm{DA} / 30 \% \mathrm{EPOX}$ ) containing $\mathrm{AgNO}_{3}$ initiated by dyes $1-5 / \mathrm{Iod} /$ amine upon exposure to a LED@405nm in laminate (c) of EPOX of IPN with the thickness about $0.1 \mathrm{~mm}$; (d) of PEG-DA of IPN with the thickness about 0.1mm; (e) of EPOX of IPN with the thickness about $2 \mathrm{~mm}$; (f) of PEG-DA of IPN with the thickness about $2 \mathrm{~mm}$.

\begin{tabular}{|c|c|c|c|c|}
\hline \multirow{2}{*}{ dye } & \multicolumn{2}{|c|}{ Thin } & \multicolumn{2}{c|}{ Thick } \\
\cline { 2 - 5 } & EPOX(\%) & PEG-DA(\%) & EPOX(\%) & PEG-DA(\%) \\
\hline 1 & 36 & 35 & 85 & 95 \\
\hline 2 & 33 & 66 & 76 & 97 \\
\hline 3 & 75 & 44 & 73 & 99 \\
\hline 4 & 52 & 26 & 96 & 98 \\
\hline 5 & 84 & 94 & 90 & 96 \\
\hline
\end{tabular}

Table 5. Summary of the final function conversions of PEG-DA and EPOX monomers using dye/iodine/amine-based PIS to 70\% PEG-DA/30\% EPOX mixed monomer (thickness about $20 \mu \mathrm{m}$ and $2 \mathrm{~mm}$ ) under LED@405nm irradiation.

\subsection{Characterization of IPN Polymers.}

Judging from the above results about the photopolymerization ability for mixed monomers, the dye 5-based three-component PIS achieved the highest efficiency, so that the dye 5 /Iod/EDB has been selected as the PIS to study the mechanical properties of IPNs. At first, a series of mixed solutions with different components (pure PEG-DA monomer, different ratio of PEG-DA/EPOX mixed monomers, mixed monomers containing $\mathrm{AgNO}_{3}$, pure EPOX monomers) were prepared; and then using a time scanning rheometer to detect these pre-polymerization formulations' deep-curing speed. Since the PEG material exhibited a volume shrinkage after it transforms from the liquid phase into the solid phase during the light irradiation process, the curves appeared disordered after photopolymerization $[47,48]$. As shown in Figure 7a, the pure 
PEG-DA monomer polymerized the fastest, while for mixed monomers, the curing speed gradually slowed down as the content of PEG-DA decreased. Addition of $\mathrm{Ag}^{+}$ also significantly decreased the curing speed of mixed monomers. More interestingly, when the EPOX content is higher than $70 \%$, the volume of the obtained polymers didn't shrink, this may be due to the lower PEG-DA content that has less effect on the volume. In addition, there is no gelation point and obvious phase inversion phenomenon observed with a litter amount of pre-polymerization EPOX formulations, which simply become more viscous as the G" was grown with light irradiation (see Figure S6). This is mainly due to the following reasons: firstly, the polymerization efficiency of pure EPOX is not very well; secondly, the optical density of the light source for the rheometer used is less than that of real-time FTIR spectroscopy.

Subsequently, a series of IPN polymers with different components were produced using molds upon visible light irradiation, and their tensile properties were measured. The results are shown in Figure 7b. Since the pure PEG-polymers is brittle, the tensile property was very poor. For the IPN-polymers, high viscosity of EPOX greatly enhances their tensile property, in which the polymer obtained by the mixed monomer composed of $70 \%$ PEG-DA/30\%EPOX exhibited the best tensile performance, that is, the elongation increases from $5 \%$ to $15 \%$ and the fracture potential energy increased from 0.55 $\mathrm{MPa}$ to about 1.2 $\mathrm{MPa}$. Adding some $\mathrm{AgNO}_{3}$ to the precursor solution would reduce the tensile properties of the IPN-polymer. The above results also show that the mixture of monomers composed of 70\%PEG-DA/30\%EPOX was the best ratio, which not only had an excellent photopolymerization ability, but also the polymer obtained by photopolymerization exhibited the best mechanical properties. 

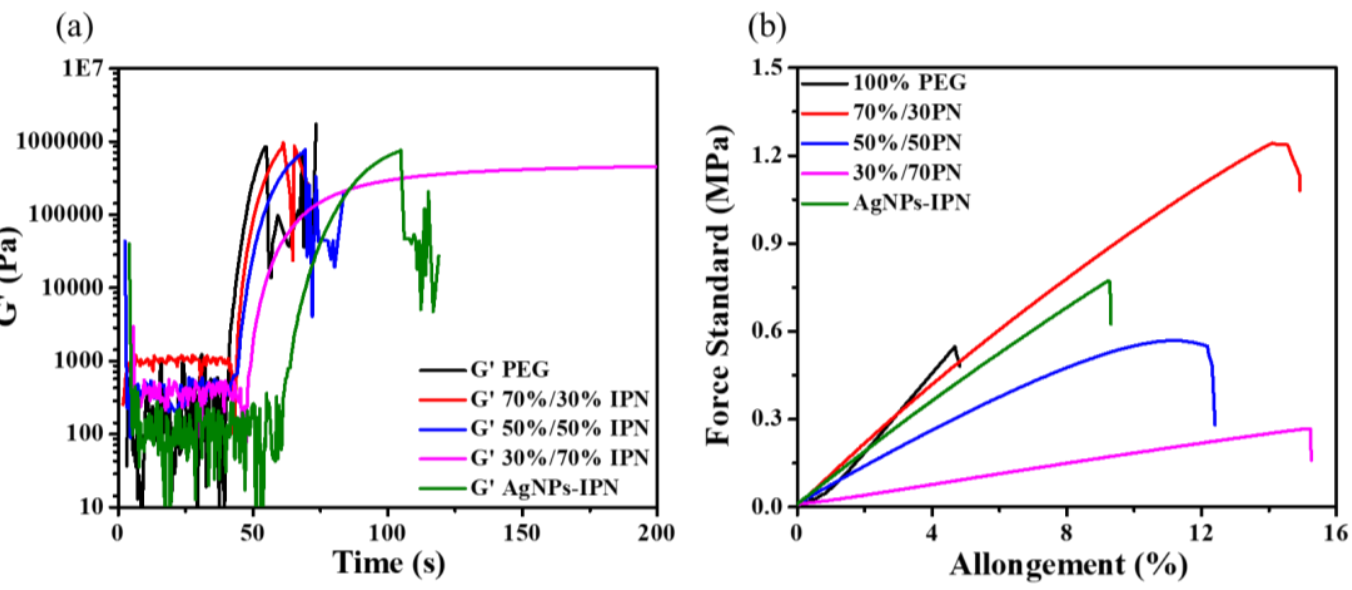

Figure 8. (a) Time sweep of photopolymerization process (irradiation starts at $t=30 \mathrm{~s}$, volume is about $40 \mu \mathrm{L}$ ) of the pure PEG-DA monomer, different ratio of PEGDA/EPOX mixed monomers as well as the mixed monomers containing $\mathrm{AgNO}_{3}$; (b) Tensile property of PEG-polymer, IPN-polymers as well as AgNPs contained IPNpolymers.

\subsection{Applications in 3D and 4D.}

3.5.1 3D laser writing experiments based on the dye 5-based three-component PISs.

Since dye 5 was selected as the best candidate, pure PEG-DA, pure EPOX, IPN, and AgNPs containted IPN with dye 5/Iod/EDB as PIS were prepared to perform laser writing experiments under visible light irradiation. These four formulations were successfully written to obtain stable 3D cross patterns ("+") with excellent spatial resolution. Among them, the fastest writing speed was achieved for pure PEG-DA resins, and a complete 3D pattern could be obtained within $1 \mathrm{~min}$. Conversely, longer irradiation times were required for IPNs and AgNPs containing IPNs to get the 3D patterns compared to that required for pure PEG resins. Writing speed of EPOX was the slowest, taking around 5 minutes. Furthermore, morphology of the obtained 3D patterns was observed with a digital optical microscope.

As shown in Figure 9, morphology of the obtained pure PEG based 3D pattern was the best, the surface of it was smooth, and the overall thickness was uniform, about 2.4 mm (see Figure 9a). For the cross pattern of pure EPOX, its shape was incomplete, that is, the lower end of the cross was missing and the thickness was uneven, which about 1.7-1.9 mm (see Figure 9b). The shape of the IPN cross pattern was complete, but the surface was not smooth enough. There were partial collapses at the upper and lower 
ends, while the middle was relatively uniform with the thickness about $2.2 \mathrm{~mm}$ (see Figure 9c). AgNPs containing IPN cross pattern was darker than the other three polymer patterns, whose surface wasn't very smooth and the thickness is about $2 \mathrm{~mm}$, which was decreased comparing to IPN pattern (see Figure 9d).
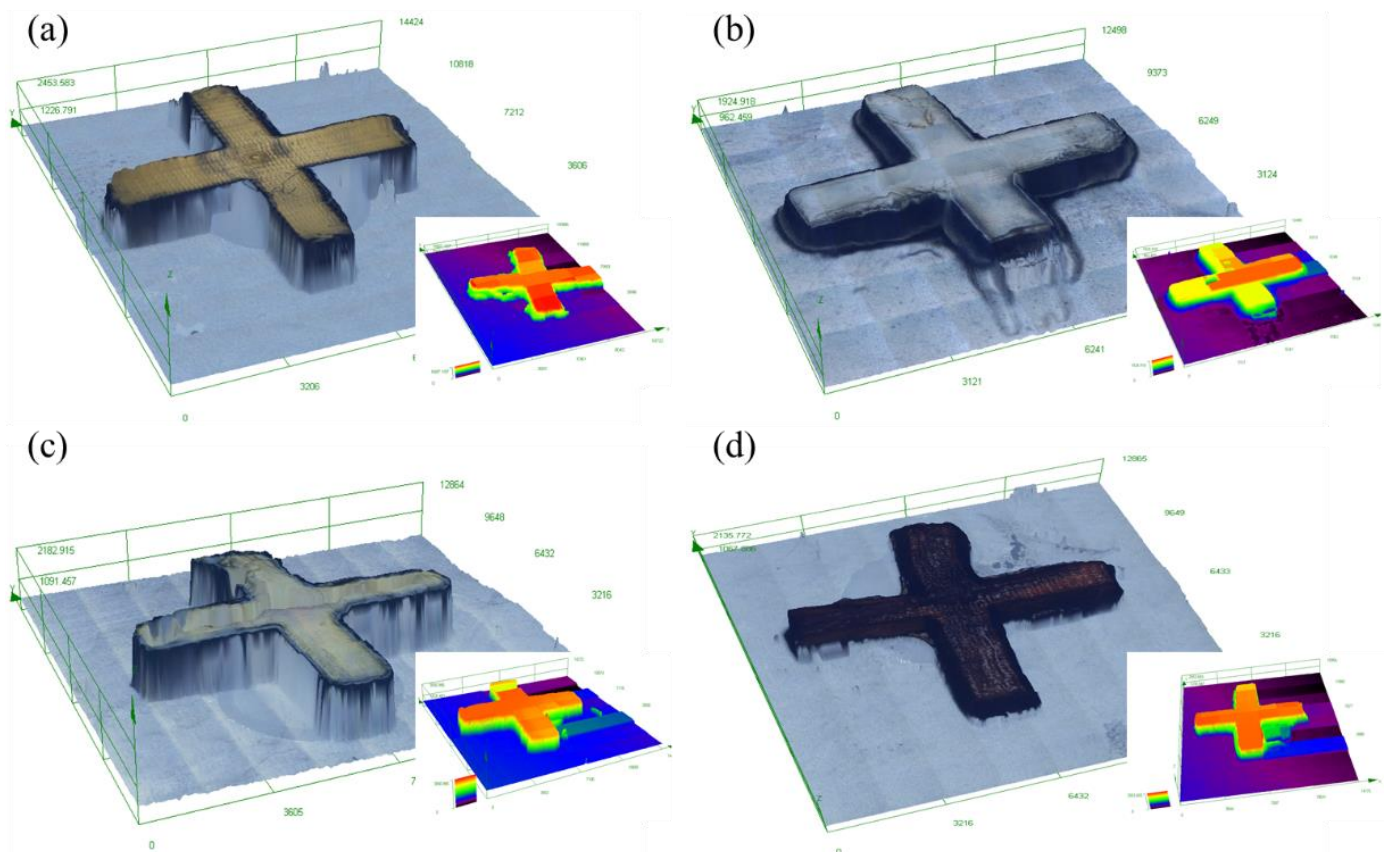

Figure 9. Characterization of the 3D overall appearance of the 3D patterns produced by the laser writing experiment through a numerical optical microscope. (a) for PEGpolymer (b) for EPOX-polymer; (c) for IPN polymer (d) for AgNP containing IPN polymer.

\subsubsection{Swelling experiment of PEG-, EPOX-, IPN- and AgNP containing IPN- polymer}

As we all know that PEG-DA is a hydrophilic material while EPOX is a hydrophobic material, the hydrophilic properties of the four kinds of polymers obtained through laser writing experiments were studied by immersing them in deionized water and comparing their weight and morphological changes. At first, the initial wet weight of the four polymers were compared with that after reaching the swelling equilibrium to calculate the swelling ratio, the result is shown in Figure 10. The pure PEG polymer exhibited the best hydrophilicity, and the swelling rate is about $80 \%$; while the volume and weight of pure EPOX polymer didn't changed as the EPOX is hydrophobic. For the IPN polymer, as the existence of EPOX hydrophobic material reduces the 
hydrophilicity of IPN polymer, its swelling ratio decreased to $60 \%$. Furthermore, introduction of AgNPs into IPN also decreased their hydrophilic character, which achieved about $40 \%$ swelling ratio. Except the hydrophobic polymer EPOX, as the weight of the polymers increased, their morphology and volume also changed significantly during the swelling process. Compared with their initial morphologies, the polymers became bigger after reaching the swelling equilibrium (see Figure 10b) and the volumes also increases (see Table 5). The volume of pure PEG, IPN and AgNPs containing IPN polymer increased about 199\%, 166\% and 138\%, respectively, which exactly matches with their swelling rates. Subsequently, the absorbed water in the polymers could be removed by dehydration to restore their original weight and morphology. However, the color of them become lighter after the swelling process $[49,50]$.

(a)

(b)
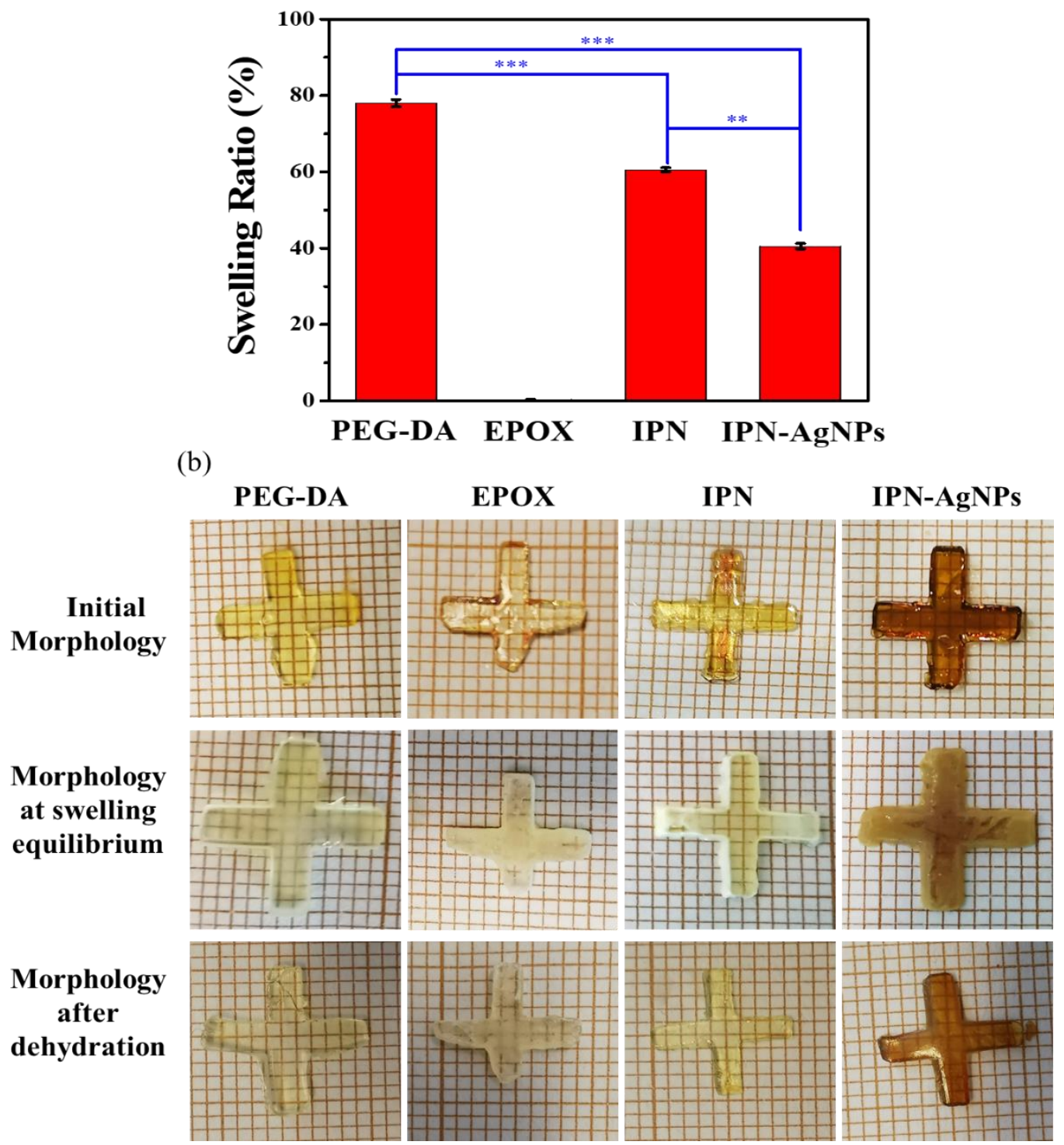
Figure 10. (A) the swelling ratio of PEG-, EPOX-, IPN- and AgNPs contained IPNpolymers initiated by dye 5/Iod/amine; (B) the photos of them during the swelling process: initial morphology, morphology at swelling equilibrium and morphology after dehydration.

\begin{tabular}{|c|c|c|c|c|}
\hline & PEG-DA & EPOX & IPN & IPN-AgNPs \\
\hline $\mathbf{V}_{\mathbf{1}}\left(\mathbf{m m}^{\mathbf{3}}\right)$ & 51.0 & 47.5 & 47.0 & 44.3 \\
\hline $\mathbf{V}_{\mathbf{2}}\left(\mathbf{m m}^{\mathbf{3}}\right)$ & 101.6 & 47.6 & 78.2 & 61.0 \\
\hline $\mathbf{V}_{\mathbf{3}}\left(\mathbf{m m}^{\mathbf{3}}\right)$ & 54.3 & 47.5 & 46.8 & 45.6 \\
\hline $\mathbf{R}(\mathbf{\%})^{\mathbf{b}}$ & 199.2 & 0 & 166.4 & 137.7 \\
\hline
\end{tabular}

${ }^{\mathrm{a}} S_{r}=\left(\mathrm{m}_{2}-\mathrm{m}_{1}\right) / \mathrm{m}_{1} * 100 \% \quad{ }^{\mathrm{b}} R=\mathrm{V}_{2} / \mathrm{V}_{1} * 100 \%[27,35,49]$

Table 4. Volumes of the obtained 3D patterns during swelling process: initial volume $\left(\mathrm{V}_{1}\right)$, volumes at swelling equilibrium $\left(\mathrm{V}_{2}\right)$, volume after dehydration $\left(\mathrm{V}_{3}\right)$ as well as the volume change ratio $(R)$.

\subsubsection{The reversible deformation effect of PEG-, IPN- and AgNP containing IPN- polymer.}

Based on the swelling properties of these four polymers, pure PEG, IPN and AgNPs-IPN polymers were used to study their reversible deformation effects. The best light irradiation time was selected by comparing the influence of ethe irradiation time to prepare the 3D cross samples, and then their 4D deformation effects were studied through swelling and dehydration processes [51-53]. As shown in Figure 11(a) and SI Video $1-2$, the shortest time ( $\sim 30$ s) was taken to prepare the pure PEG sample, which is yellow and transparent. It is completely deformed after being swelled in water for 1 min, and then dehydrated in an oven at $80^{\circ} \mathrm{C}$ for $6 \mathrm{~min}$ to restore its original appearance. While for the second deformation process, even though it only took $15 \mathrm{~s}$ to swell again to achieve a complete deformation, it still dehydrated for 6 min to recover its initial appearance. When the dehydration of PEG sample was continued, a reverse curling can be observed. For the IPN polymer shown in Figure 11(b) and SI Video 1-2, the preparation time was also short, about $45 \mathrm{~s}$ and the polymer was yellow and opaque. Driven by swelling and dehydration, it also exhibited a reversible deformation effect, 
but due to its hydrophilic property which was lower than that of pure PEG polymer, its deformation process was faster that can be finished within $2 \mathrm{~min}$, and no reverse curling effect was observed with continuous heating. After introducing AgNPs into IPN polymers, it took a long time $(3 \mathrm{~min})$ to produce the samples which is brown-yellow. The reversible 4D deformation effect can also be observed through repeated swelling and dehydration processes, and the time required was shorter which may be due to its lowest polymerization efficiency which leads in turn to a looser polymerization network and easier deformation. In addition, continued dehydration only deepens the color of the polymer. The above results indicate that the $4 \mathrm{D}$ deformation effect caused by the thermal response and hydrophilic responsiveness of the IPN polymer is better than that of the pure PEG polymer, but after the introduction of metal nanoparticles, although the sample preparation time was prolonged, the quality of the obtained polymer sample was reduced. However, effect of its 4D deformation has not been greatly affected. 
(a)

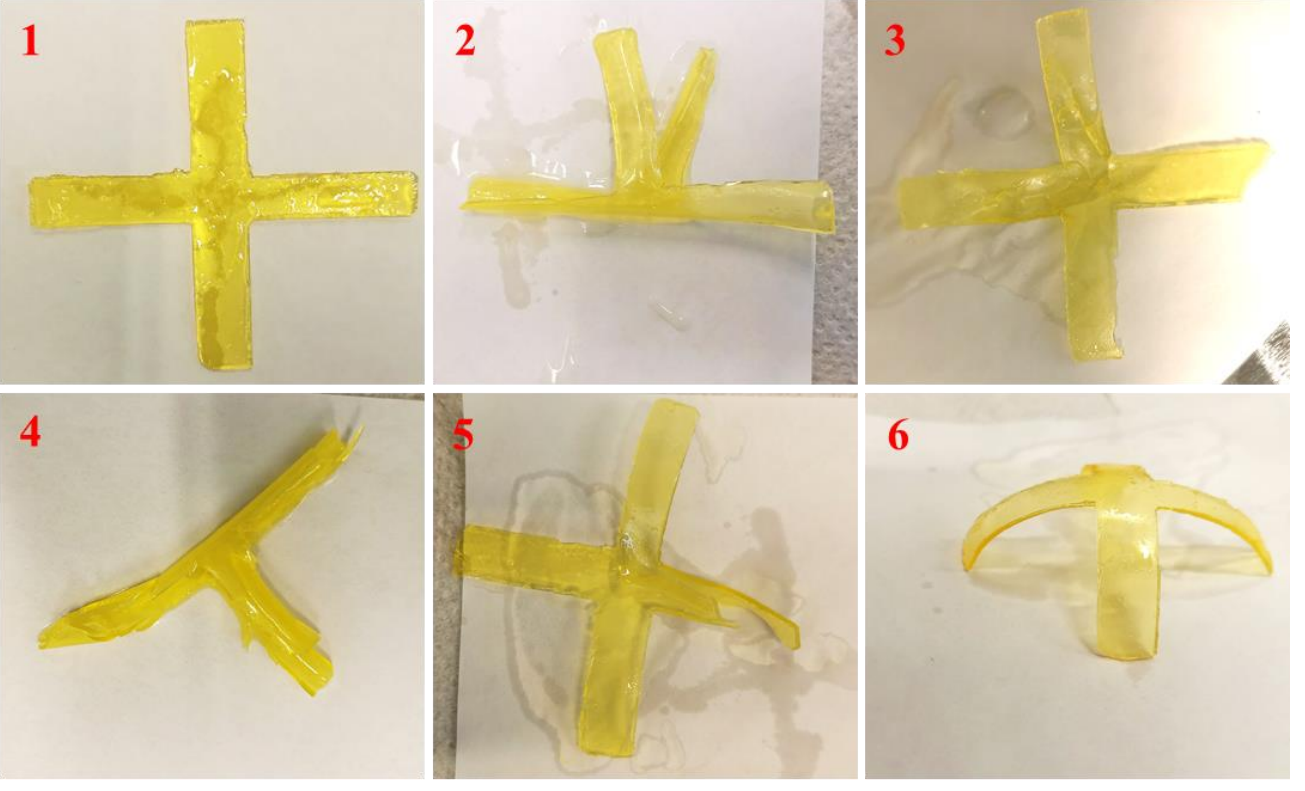

(b)

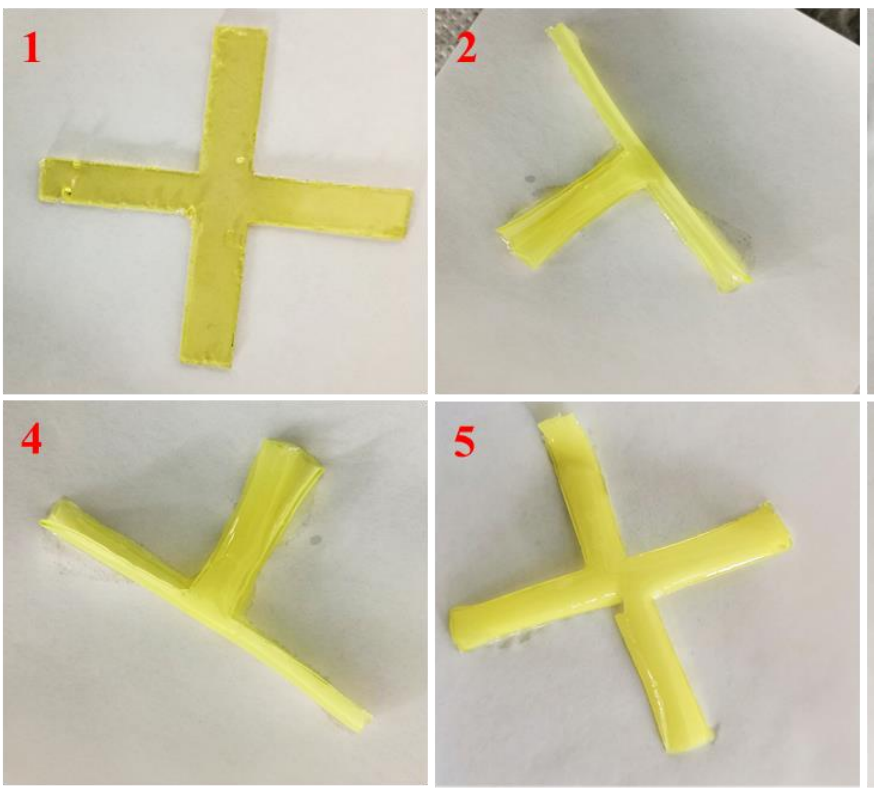

(c) 1

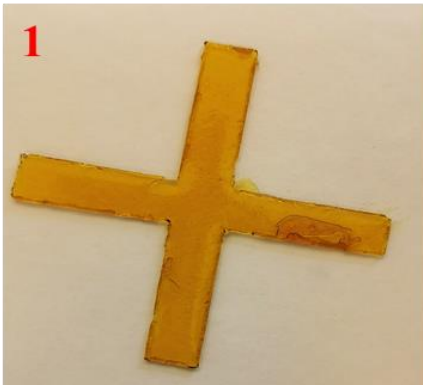

4

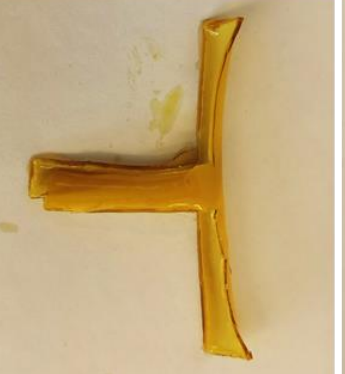

2

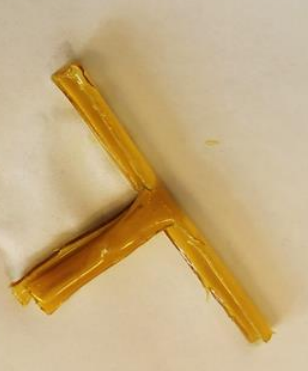

5

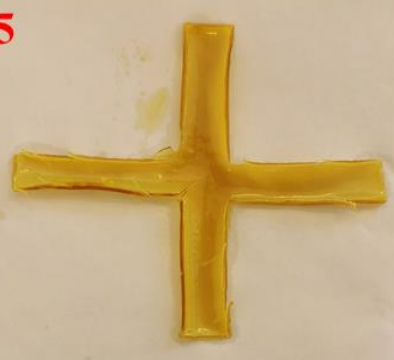

3

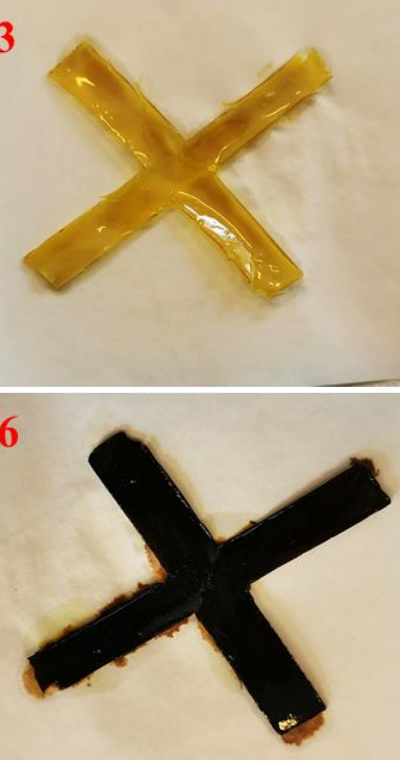


Figure 11. Swelling and dehydration induced reversible deformation effect of (a) PEGpolymer, (b) IPN-polymer and (c) AgNPs contained IPN-polymer initiated by dye 5/Iod/amine upon irradiation with LED@405 nm: (1) photos of 3D cross polymers prepared with the visible light irradiation; (2) photos of 3D cross polymers after swelling in water for different time (see SI Video 1/3/5); (3) photos of 3D cross polymers after dehydration for a few time (heating at $80^{\circ} \mathrm{C}$, see SI Video $1 / 3 / 5$ ); (4) photos of 3D cross polymers after swelling again in water for some time (see SI Video 2/4/6); (5) photos of 3D cross polymers after dehydration again for $100 \mathrm{~s}$ (heating at 80 ${ }^{\circ} \mathrm{C}$, see SI Video 2/4/6); (6) photos of 3D cross polymers after continue dehydration for $5 \mathrm{~min}$ (heating at $80^{\circ} \mathrm{C}$ ).

\subsection{Antibacterial effect of IPN containing/without AgNPs.}

As plenty of researches have proved that AgNPs have a broad range of antibacterial activities, which represent a new generation of antimicrobials [54-57]. Therefore, in this work, the Gram-positive bacteria Staphylococcus aureus and the Gram-negative bacteria Pseudomonas aeruginosa were selected to study the antibacterial effect of the fabricated AgNPs contained IPN polymer.

Three types of experiments were carried out:

First, cultures of $S$. aureus and P. aeruginosa was perform in liquid LB and LBagar in the presence of IPN alone and in presence of AgNPs containing IPN. After one night at $37^{\circ} \mathrm{C}$ in agitation the comparison by eyes of the Erlen flasks (Figure 12) show clearly that no turbidity is visible when the AgNPs-IPN is present whereas a normal growth (final OD at approximately 3 ) is detectable when IPN alone is present. In the solid medium, a halo of growth inhibition is also observable only around the AgNPsIPN. Secondly, in order to check if the effect of the presence of the AgNPs-IPN is bacteriostatic or bacteriotytic, $100 \mu \mathrm{L}$ of each culture was spread on LB Petri dishes to obtain CFU. With S. aureus, no colony were obtained whereas in the IPN control the same $3.10^{9} \mathrm{CFU}$ (colony forming unit = bacteria $/ \mathrm{mL}$ ) were counted as in the LB control. Therefore, the presence of the AgNPs-IPN has killed the S. aureus cells. With $P$. aeruginosa, the effect is less drastic because the spreading of $100 \mu \mathrm{L}$ from the AgNPs- 
IPN containing Erlen show the formation of bacterial layer covering the Petri dishes. Therefore, the antibacterial effect on the Gram-negative strain seems only bacteriostatic. Finally, we also test the capacity of both bacterial species to adhere to the polymer. Logically no adhesion was observed for S. aureus on the AgNPs-IPN in comparison of the $10^{4}+/-100$ adherent cells on the IPN. $70+/-10$ cells of $P$. aeruginosa were retrieved form the AgNPs-IPN, and $10^{5}+/-100$ cells were adherent to the IPN. Adhesion tests confirm the precedent results.

(a)

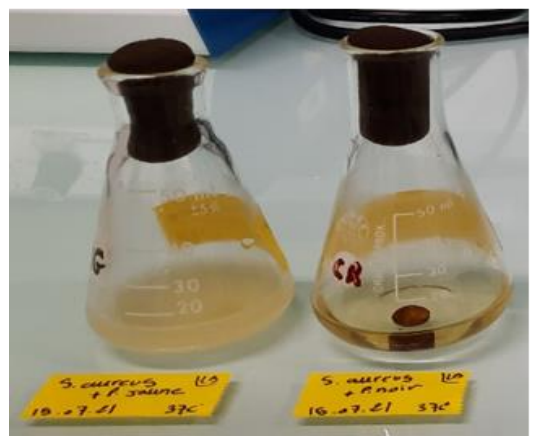

(c)

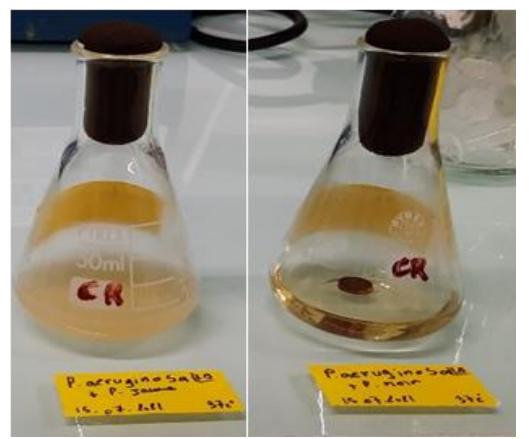

Fugure 12. (a) Effect of the presence of IPN pellet on the growth of S. aureus; (b) Effect of the presence of AgNPs contained IPN pellet on the growth of S. aureus; (c) Effect of the presence of IPN pellet on the growth of P. aeruginosa; (d) Effect of the presence of AgNPs contained IPN pellet on the growth of P. aeruginosa.

\section{Conclusions}

In this work, eleven different dyes based on 1-aryl-3-(2,4,5trimethoxyphenyl)prop-2-en-1-one or 3-aryl-1-(3,4,5-trimethoxyphenyl)prop-2-en-1one were synthesized and combined with Iod and EDB as visible light PIS. Under LED@405nm irradiation, the redox reaction between the dye and Iod/EDB generates a series of free radicals and cations to initiate the polymerization of PEG-DA and EPOXY monomers, in which dye 5 was selected as the best candidate for the preparation of IPN polymers with different proportions. According to the photopolymerization efficiency and mechanical properties of different IPN polymers, the mixed monomer composed of 70\%PEG-DA/30\%EPOX has proved to be the best ratio. The mechanical strength of the obtained IPN polymer has been greatly improved, demonstrating that compared with a single network, the IPN exhibits its unique performance due to the combination of multiple monomers in terms of physical and chemical properties. Due to the 
hydrophilicity of PEG and the hydrophobicity of EPOX, the IPN polymer exhibits a reversible deformation effect that is different from that of pure PEG polymer. In addition, the three-component PIS based on the dye 5/Iod/EDB can not only induce the photopolymerization reaction, but also reduce the metal salt to AgNPs. However, the formation of AgNPs will darken the color of the polymer, meanwhile it also exhibited some inhibitory effect on photopolymerization process, making the physicochemical properties of the polymer inferior to IPN. Remarkably, the synthetic dye-based photoinitiator combines free radical polymerization and cationic polymerization to prepare an IPN polymer that containing the hydrophilicity and hydrophobicity of the two resins. In addition, the silver nanoparticles generated by in situ reduction during the photopolymerization process exhibited excellent antibacterial activity for S. aureus and P. aeruginosa, providing new ideas for the fields of biomedical engineering, intelligent response and antibacterial materials.

\section{Supplementary Materials:}

The following are available online

Acknowledgments: This research project is supported by China Scholarship Council (CSC) 201906280059. P. X. acknowledges funding from the Australian Research Council (FT170100301). This work was granted access to the HPC resources of the Mesocentre of the University of Strasbourg. This research was also funded by the Agence Nationale de la Recherche (ANR agency) through the PhD grant of Nicolas Giacoletto (ANR-19-CE07-0042, NOPEROX project).

\section{Conflicts of Interest:}

The authors declare no conflict of interest.

\section{References}

1. Sperling, L.H. Interpenetrating Polymer Networks: An Overview. In Interpenetrating Polymer Networks, Editor(s): Klempner, D.; Sperling, L.H.; Utracki, L.A. Amer. Chem. Soc. Washington, 1994, 239, 3-38. 
2. Kaneyoshi, A.; Kadzuo, I. Chapter 2: Thermosetting Forms. Editor(s): Landrock, A.H. Handbook of Plastic Foams, William Andrew Publishing. 1995, 11-220.

3. Hasan, M.; Habib, L.; Anwaruzzaman, Md.; Kamruzzaman, Md.; Nuruzzaman Khan, N.; Rahman, M.M. Chapter 3: Processing Techniques of Chitosan-Based Interpenetrating Polymer Networks, Gels, Blends, Composites and Nanocomposites. Editor(s): Gopi, S.; Thomas, S.; Pius, A. Handbook of Chitin and Chitosan, Elsevier, 2020, 61-93.

4. Ha, S.M.; Yuan, W.; Pei, Q.B.; Pelrine, R.; Stanford, S. Chapter 5: Interpenetrating Polymer Networks as High Performance Dielectric Elastomers, Editor(s): Carpi, F.; Rossi, D.D.; Kornbluh, R.; Pelrine, R.; Sommer-Larsen, P. Dielectric Elastomers as Electromechanical Transducers, Elsevier, 2008, 43-50.

5. Munsur, A.Z.A.; Goo, B.H.; Kim, Y.; Kwon, O.J.; Paek, S.Y.; Lee, S.Y.; Kim, H.J.; Kim, T.H. Nafion-Based Proton-Exchange Membranes Built on Cross-Linked Semi-Interpenetrating Polymer Networks between Poly (acrylic acid) and Poly (vinyl alcohol). ACS Appl. Mater. Interfaces. 2021, 13(24), 28188-28200.

6. Roland C.M. Interpenetrating Polymer Networks (IPN): Structure and Mechanical Behavior. Editor(s): Kobayashi S., Müllen K. Encyclopedia of Polymeric Nanomaterials. Springer, Berlin, Heidelberg. 2013.

7. Alizadeh, N.; Farag, R.; Auad, M. Flexible Graft-Interpenetrating Polymer Networks Consisting of Polyurethane and Acrylic Copolymer for Transparent, High-Performance Applications. 2020.

8. Pour-Esmaeil, S.; Qazvini, N.T.; Mahdavi, H. Interpenetrating Polymer Networks (IPN) based on Gelatin/Poly (Ethylene Glycol) Dimethacrylate/Clay Nanocomposites: Structure-Properties Relationship. Mater. Chem. Phys. 2014, 143(2), 1396-1403.

9. Sundaram, B.M.; Mendez, R.B.; Auad, M.L.; Tippur, H.V. Quasi-Static and Dynamic Mechanical Behavior of Transparent Graft-Interpenetrating Polymer Networks (Graft-IPNs). Polym. Test. 2018, 70, 348-362.

10. Alizadeh, N.; Bird, S.A.; Mendez, R.B.; Jajam, K.C.; Alexander, A.C.; Tippur, H. V.; Auad, M.L., Chapter 11: Synthesis and Characterization of High Performance Interpenetrating Polymer Networks with Polyurethane and Poly (methyl methacrylate). In Unsaturated Polyester Resins; Editor(s): Thomas, S.; Hosur, M.; Chirayil, C.J. Elsevier, 2019, 243-255.

11. Sundararajan, S.; Samui, A.B.; Kulkarni, P.S. Interpenetrating Phase Change 
Polymer Networks Based on Crosslinked Polyethylene Glycol and Poly (Hydroxyethyl Methacrylate). Sol. Energy. Mater. Sol. Cells. 2016, 149, 266-274.

12. Maity, S.; Chatterjee, A.; Ganguly, J. Chapter 18: Stimuli-Responsive SugarDerived Hydrogels: A Modern Approach in Cancer Biology. Editor(s): Banik, K.B. In Advances in Green Chemistry, Green Approaches in Medicinal Chemistry for Sustainable Drug Design. Elsevier, 2020, 617-649.

13. Yu, F.; Yang, P.Y.; Yang, Z.Q.; Zhang, X.C.; Ma, J. Double-Network Hydrogel Adsorbents for Environmental Applications. Chem. Eng. J. 2021, 131900.

14. Aminabhavi, T.M.; Dharupaneedi, S.P. Chapter 12: Production of Chitosan-based Hydrogels for Biomedical Applications. Editor(s): Jennings, J.A.; Bumgardner, J.D. Chitosan Based Biomaterials, Woodhead Publishing, 2017, 1, 295-319.

15. Sophiea, D.; Klempner, D.; Sendijarevic, V.; Suthar, B.; Frisch K.C. Interpenetrating Polymer Networks as Energy-Absorbing Materials. Interpenetrating Polymer Networks. 1994, 39-75.

16. Smith, G.N.; Brok, E.; Schmiele, M.; Mortensen, K.; Bouwman, W.G.; Duif, C.P.; Hassenkam, T.; Alm, M.; Thomsen, P.; Arleth, L. The Microscopic Distribution of Hydrophilic Polymers in Interpenetrating Polymer Networks (IPNs) of Medical Grade Silicone. Polymer. 2021, 123671.

17. Ma, Y.F.; Ji, Y.; Zhong T.Y.; Wan, W.T.; Yang, Q.Z.; Li, A.; Zhang, X.H.; Lin, M.;

Bioprinting-based PDLSC-ECM Screening for in Vivo Repair of Alveolar Bone Defect using Cell-Laden, Injectable and Photo-Crosslinkable Hydrogels. ACS Biomater. Sci. Eng. 2017, 12, 3534-3545.

18. Schesny, M.K.; Monaghan, M.; Bindermann, A.H.; Freund, D.; Seifert. M.; Eble, J.A.; Vogel, S.; Gawaz, M.P.; Hinderer, S.; Schenke-Layland, K. Preserved Bioactivity and Tunable Release of a SDF1-GPVI Bi-Specific Protein using PhotoCrosslinked PEGDA Hydrogels. Biomaterials 2014, 35(25), 7180-7187.

19. Liu, S.; Yeo, D.C.; Wiraja, C.; Tey, H.L.; Mrksich, M.; Xu, C. Peptide Delivery with Poly (Ethylene Glycol) Diacrylate Microneedles through Swelling Effect. Bioeng. Transl. Med. 2017, 2(3), 258-267.

20. Hutson, C.B.; Nichol, J.W.; Aubin, H.; Bae, H.; Yamanlar, S.; Al-Haque, S.; Koshy, S.T.; Khademhosseini, A. Synthesis and Characterization of Tunable Poly (Ethylene Glycol): Gelatin Methacrylate Composite Hydrogels. Tissue. Eng. Part A. 2011, 17(13-14), 1713-1723. 
21. Guarino, V.; Alvarez-Perez, M.A.; Borriello, A.; Napolitano, T.; Ambrosio, L. Conductive PANi/PEGDA Macroporous Hydrogels for Nerve Regeneration. Adv. Healthcare. Mater. 2013, 2(1), 218-227.

22. Zeng, Z. Mo, X.M.; He, C.; Morsi, Y.; El-Hamshary, H.; El-Newehy, M. An in situ Forming Tissue Adhesive based on Poly (Ethylene Glycol)-Dimethacrylate and Thiolated Chitosan through the Michael Reaction. J. Mater. Chem. B. 2016, 4(33), 5585-5592.

23. Nachlas, A.L.; Li, S.; Jha, R.; Singh, M.; Xu, C.; Davis, M.E. Human iPSC-derived Mesenchymal Stem Cells Matured into Valve Interstitial-like Cells using PEGDA Hydrogels. Acta. Biomater. 2018, 11(1), 015019.

24. Chen, H.; Noirbent, G.; Liu, S.H.; Brunel, D.; Graffa, B.; Gigmes, D.; Zhang, Y.J.; Sun, K.; Morlet-Savary, F.; Xiao, P.; Dumur, F.; Lalevée, J. Bis-Chalcone Derivatives Derived from Natural Products as Near-UV/Visible Light Sensitive Photoinitiators for 3D/4D Printing. Mater. Chem. Front. 2021, 5, 901-916.

25. Chen, H.; Noirbent, G.; Liu, S.H.; Zhang, Y.J.; Sun, K.; Morlet-Savary, F.; Gigmes, D.; Xiao, P.; Dumur, F.; Lalevée, J. In Situ Generation of Ag Nanoparticles during Photopolymerization by using Newly Developed Dyes-based Three-Component Photoinitiating Systems and the Related 3D Printing Applications and Their Shape Change Behavior. J. Polym. Sci. 2021, 59 (10), 843-859.

26. Zhang, J.; Wang, S.H.; Lalevée, J.; Morlet-Savary, F.; Lam, E.S.H.; Graff, B.; Liu, J.; Xing, F.Y.; Xiao, P. 1,2-Diketones as Photoinitiators of both Cationic and FreeRadical Photopolymerization under UV (392 nm) or Blue (455 nm) LEDs. J. Polym. Sci. 2020, 58, 792-802.

27. Chen, H.; Noirbent, G.; Zhang, Y.J.; Sun, K.; Liu, S.H.; Brunel, D.; Gigmes, D.; Graffa, B.; Morlet-Savary, F.; Xiao, P.; Dumur, F.; Lalevée, J. Photopolymerization and 3D/4D Applications using Newly Developed Dyes: Search Around the Natural Chalcone Scaffold in Photoinitiating Systems. Dyes and Pigments. 2021, 188, 109213.

28. Debdatta R.; Dalvi, V.G.; Billa, S.; Sharma, S.K.; Rath, S.K.; Sudarshan, K.; Pujari, P.K. Interpenetrating Polymer Network of Rubbery Epoxy and Glassy PMMA: Network Inhomogeneities and Dynamic Heterogeneities. ACS Appl. Polym. Mater. 2021.

29. Zhao, X.H.; Chen, X.Y.; Yuk, H.; Lin, S.T.; Liu, X.Y.; Parada, G. Soft Materials by Design: Unconventional Polymer Networks Give Extreme Properties. Chem. 
Rev. 2021, 121(8), 4309-4372.

30. Alizadeh, N.; Triggs, E.; Farag, R.; Auad, M. Flexible Acrylic-Polyurethane based Graft-Interpenetrating Polymer Networks for High Impact Structural Applications. Eur. Polym. J. 2021, 148, 110338.

31. Zhang, J.; Hill, N.; Lalevee, J.; Fouassier, J.P.; Zhao, J.; Graff, B.; Schmidt, T.W.; Kable, S.H.; Stenzel, M.H.; Coote, M.L.; Xiao, P. Multihydroxy-Anthraquinone Derivatives as Free Radical and Cationic Photoinitiators of Various Photopolymerizations under Green LED. Macromol. Rapid. Commun. 2018, 39, e1800172.

32. Xiao, P.; Zhang, J.; Dumur, F.; Tehfe, M.-A.; Morlet-Savary, F.; Graff, B.; Gigmes, D.; Fouassier, J.-P.; Lalevée, J. Photoinitiating Systems: Recent Progress in Visible Light Induced Cationic and Radical Photopolymerization Reactions under Soft Conditions. Prog. Polym. Sci. 2015, 41, 32-66.

33. Duling, D.R. Simulation of Multiple Isotropic Spin-Trap EPR Spectra. J. Magn. Reson. 1994, 104(2), 105-110.

34. Aleksandra, S.; Murielle, O.; Marine, D.; Loïc, V.; Lavinia, B. Photo-Induced Design of Reflective Metallized Gold@Polymer Coatings with Tuned Architecture. Mater. Des. 2018, 160, 74-83.

35. Chen, H.; Zhang, Y.J.; Ni, T.Y.; Ding, P.; Zan, Y.; Cai, X.; Zhang, Y.W.; Liu, M.; Pei, R.J. Construction of a Silk Fibroin/Polyethylene Glycol Double Network Hydrogel with Co-Culture of HUVECs and UCMSCs for a Functional Vascular Network. ACS Appl. Bio. Mater. 2021, 4(1), 406-419.

36. Chen, H.; Vahdati, M.; Xiao, P.; Dumur, F.; Lalevée, J. Water-Soluble Visible Light Sensitive Photoinitiating System Based on Charge Transfer Complexes for the 3D Printing of Hydrogels. Polymers. 2021. 
37. Zhang, Z.H.; Corrigan, N.; Bagheri, A.; Jin, J.Y.; Boyer, C.A Versatile 3D and 4D Printing System through Photocontrolled RAFT Polymerization. Angewandte Chemie. 2019, 131, 18122-18131.

38. Liu, Y.; Li, Y.; Yang, G.; Zheng, X.T.; Zhou, S.B. Multi-Stimulus-Responsive Shape-Memory Polymer Nanocomposite Network Cross-Linked by Cellulose Nanocrystals. ACS. Applied. Materials \& Interfaces. 2015, 7 (7), 4118-4126.

39. Lalevée, J.; Rahal, M.; Mokbel, H.; Graff, B.; Pertici, V.; Gigmes, D.; Toufaily, J.; Hamieh, T.; Dumur, F. New Naphthalimides as Photoinitiators under Visible Light Irradiation and their Application: Photocomposite Synthesis, 3D Printing and Polymerization in Water. Chem. Photo. Chem. 2021.

40. Chen, H.; Noirbent, G.; Zhang, Y.J.; Brunel, D.; Gigmes, D.; Liu, S.H.; Sun, K.; Morlet-Savary, F.; Graff, B.; Xiao, P.; Dumur, F.; Lalevée, J. Novel D- $\pi$-A and A$\pi$-D- $\pi$-A Three-Component Photoinitiating Systems Based on Carbazole/Triphenylamino based Chalcones and Application in 3D/4D Printing. Polymer Chemistry. Polym. Chem. 2020, 11, 6512-6528.

41. Liang, Z.Q.; Wang, X.M.; Dai, G.L.; Ye, C.Q.; Zhou, Y.Y.; Tao, X.T. The Solvatochromism and Aggregation-Induced Enhanced Emission based on Triphenylamine-Propenone. New. J. Chem. 2015, 39(11), 8874-8880.

42. Liu, S.H.; Brunel, D.; Noirbent, G.; Mau, A.; Chen, H.; Morlet-Savary, F.; Graff, B.; Gigmes, D.; Xiao, P.; Dumur, F.; Lalevée, J. New Multifunctional Benzophenone-based Photoinitiators with High Migration Stability and Their Applications in 3D Printing. Mater. Chem. Front. 2021, 5, 1982-1994.

43. Fouassier, J.P.; Photoinitiator, Photopolymerization and Photocuring: Fundadamentals and Applications. New York, Munich, Vienna: Hanser Publishers, 1995, 388.

44. Rehm, D.; Weller, A. Kinetics of Fluorescence Quenching by Electron and H-Atom Transfer. Isr. J. Chem. 1970, 8, 259-271. 
45. Arsuaga, J.M.; Sotto, A.; Rosario, del G.; Martínez, A.; Molina, S.; Teli, S.B.; Abajo, de. J. Influence of the Type, Size, and Distribution of Metal Oxide Particles on the Properties of Nanocomposite Ultrafiltration Membranes. J. Membr. Sci. 2013, 428, 131-141.

46. Caillosse, E.; Zaier, M.; Mezghani, M.; Hajjar-Garreau, S.; Vidal, L.; Lougnot, D.; Balan, L. Photo-Induced Self-Assembly of Silver Nanoparticles for Rapid Generation of First and Second Surface Mirrors. ACS Appl. Nano. Mater. 2020, 3, 6531-6540.

47. Kurdikar, D.L.; Peppas, N.A. The Volume Shrinkage, Thermal and Sorption Behaviour of Polydiacrylates. Polymer 1995, 36(11), 2249-2255.

48. Löwenberg, C.; Balk, M.; Wischke, C.; Behl, M.; Lendlein, A. Shape-Memory Hydrogels: Evolution of Structural Principles to Enable Shape Switching of Hydrophilic Polymer Networks. Acc. Chem. Res. 2017, 50(4), 723-732.

49. Albers, P.T.M.; Ven, L.G.J.V.D.; Benthem, R.A.T.M.V.; Esteves, A.C.C.; Gijsbertus, D.W. Water Swelling Behavior of Poly (Ethylene Glycol)-Based Polyurethane Networks. Macromolecules 2020, 53(3), 862-874.

50. Katono, H.; Maruyama, A.; Sanui, K.; Ogata, N.; Okano, T.; Sakurai, Y. ThermoResponsive Swelling and Drug Release Switching of Interpenetrating Polymer Networks Composed of Poly (Acrylamide-co-Butyl Methacrylate) and Poly (Acrylic Acid). J. Control. Release. 1991, 16(1-2), 215-227.

51. Cao, Y.P.; Guan, Y.; Du, J.; Luo, J.; Peng, Y.X.; Yip, C.W.; Chan, A.S.C. Hydrogen-Bonded Polymer Network-Poly (Ethylene Glycol) Complexes with Shape Memory Effect. J. Mater. Chem. 2002, 12, 2957-2960.

52. Wang, Y.R.; Li, J.; Li, X.J.; Pan, Y.; Zheng, Z.H.; Ding, X.B.; Peng, Y.X. Relation Between Temperature Memory Effect and Multiple-Shape Memory Behaviors Based on Polymer Networks. RSC Adv. 2014, 4, 20364-20370.

53. Liu, G.Q.; Ding, X.B.; Cao, Y.P.; Zheng, Z.H.; Peng, Y.X. Shape Memory of Hydrogen-Bonded Polymer Network/Poly (ethylene glycol) Complexes. Macromolecules 2004, 37(6), 2228-2232.

54. Li, W.R.; Xie, X.B.; Shi, Q.S.; Zeng, H.Y.; Ou-Yang, Y.S.; Chen, Y.B. (2010) Antibacterial activity and mechanism of silver nanoparticles on Escherichia coli. Appl. Microbiol. Biotechnol. 2010, 85, 1115-1122.

55. Li, W.R.; Xie, X.B.; Shi, Q.S.; Duan, S.S.; Ouyang, Y.S.; Chen, Y.B. Antibacterial 
effect of silver nanoparticles on Staphylococcus aureus. Biometals 2011, 24, 135141.

56. Espinosa-Cristo`bal, L.F.; Martı'nez-Castaño'n, G.A.; Martı'nez-Martı'nez, R.E.; Loyola-Rodrı'guez, J.P.; Patiñ-Marı'n, N.; Reyes-Macı'as, J.F.; Ruiz, F. Antibacterial effect of silver nanoparticles against Streptococcus mutans. Materials. Letters. 2009, 63, 2603-2606.

57. Rai, M.; Yadav, A.; Gade, A. Silver nanoparticles as a new generation of antimicrobials. Biotechnol. Adv. 2009, 27, 76-83. 